January (revised May) 1998

OKHEP-97-07

UNITU-THEP-1/1998

hep-ph/9801379

\title{
Chiral Odd Structure Functions from a Chiral Soliton
}

\author{
L. Gamberg ${ }^{a)}$, H. Reinhardt ${ }^{b)}$ and H. Weigel ${ }^{b)}$ \\ ${ }^{a)}$ Department of Physics and Astronomy \\ University of Oklahoma \\ $440 \mathrm{~W}$. Brooks Ave \\ Norman, OK 73019-0225, USA \\ ${ }^{b)}$ Institute for Theoretical Physics \\ Tübingen University \\ Auf der Morgenstelle 14 \\ D-72076 Tübingen, Germany
}

\begin{abstract}
We calculate the chiral odd quark distributions and the corresponding structure functions $h_{T}\left(x, Q^{2}\right)$ and $h_{L}\left(x, Q^{2}\right)$ within the Nambu-Jona-Lasinio chiral soliton model for the nucleon. The $Q^{2}$ evolution of the twist- 2 contributions is performed according to the standard GLAP formalism while the twist-three piece, $\bar{h}_{L}(x)$, is evolved according to the large $N_{C}$ scheme. We carry out a comparison between the chiral odd structure functions of the proton and the neutron. At the low model scale $\left(Q_{0}^{2}\right)$ we find that the leading twist effective quark distributions, $f_{1}^{(q)}\left(x, Q_{0}^{2}\right), g_{1}^{(q)}\left(x, Q_{0}^{2}\right)$ and $h_{T}^{(q)}\left(x, Q_{0}^{2}\right)$ satisfy Soffer's inequality for both quark flavors $q=u, d$.
\end{abstract}

PACS: 11.30.Cp, 12.39.Ki.

To be published in: Phys. Rev. D. 


\section{Introduction}

There have been a number of recent investigations into the chiral odd structure functions of the nucleon. As in the case of the polarized structure functions there are two quantities of interest at leading twist: The transverse spin chiral odd structure function $h_{T}\left(x, Q^{2}\right)$ and the longitudinal spin chiral odd structure function $h_{L}\left(x, Q^{2}\right)$. Within the context of the operator product expansion (OPE) the analysis in terms of twist reveals that the transverse chiral odd structure function $h_{T}\left(x, Q^{2}\right)$ is purely twist-2, while the longitudinal structure function $h_{L}\left(x, Q^{2}\right)$ contains both twist-2 and twist-3 contributions. Accordingly, the decomposition of $h_{L}\left(x, Q^{2}\right)$ into twist-2 and twist-3 $\left(\bar{h}_{L}\left(x, Q^{2}\right)\right)$ pieces is given by

$$
h_{L}\left(x, Q^{2}\right)=2 x \int_{x}^{1} d y \frac{h_{T}\left(y, Q^{2}\right)}{y^{2}}+\bar{h}_{L}\left(x, Q^{2}\right) .
$$

As a reminder we note that the kinematics are defined such that $q$ denotes the momentum transferred to a nucleon of momentum $p$. In the Bjorken limit, i.e. $Q^{2}=-q^{2} \rightarrow \infty$ with $x=Q^{2} / 2 p \cdot q$ fixed, the leading twist contributions to the nucleon structure functions dominate the $1 / Q^{2}$ expansion. The additional and important logarithmic dependence on $Q^{2}$, which is associated with soft gluon emission, is included via the evolution program of perturbative quantum-chromo-dynamics (QCD).

While the chiral odd structure functions are not directly accessible in deep inelastic lepton nucleon scattering (DIS) there is the well known proposal at $R H I C$ to extract the

quark transversality distributions $h_{T}^{(a)}\left(x, Q^{2}\right)$ (a being the flavor index) from Drell-Yan dilepton-production resulting from transversely polarized proton beams [1]. Unfortunately dilepton production processes are difficult to extract from proton-proton collisions as the purely hadronic processes dominate. Furthermore this experiment will provide only the product of the chiral odd distributions for quarks and antiquarks. As the latter are presumably small these flavor distributions are not easily measurable in the Drell-Yan process. In the light of these disadvantages it has recently been pointed out that the transversality distributions may also be measured in the fragmentation region of DIS [2]. The key observation is that these distribution functions can be extracted from an asymmetry in the two meson production in the special case that this two meson state (like $\pi^{+} \pi^{-}$) is a superposition of different $C$-parity states, as e.g. $\sigma$ and $\rho$. Then the phases in the final state interactions do not vanish on the average and the differential cross section 
is proportional to the product of chiral odd distributions and the interference fragmentation functions. The latter describe the emission and subsequent absorption of a two pion intermediate state from quarks of different helicity. In case these fragmentation functions are not anomalously small the chiral odd distribution functions can then be obtained from DIS processes $\rrbracket^{\llbracket}$ like $e N \rightarrow e^{\prime} \pi^{+} \pi^{-} X$ with the nucleon $N$ being transversely polarized. Assuming isospin covariance for the fragmentation functions these DIS processes will provide access to the charge squared weighted chiral odd distribution functions [2]. Such processes should be measurable in the transversely polarized target experiments at HERMES. Knowledge of the chiral odd structure functions will serve to complete our picture of the spin structure of the nucleon as they correspond to the distribution of the quark transverse spin in a nucleon which is transversely polarized [3]. With these data being expected in the near future it is, of course, interesting to understand the structure of the nucleon from the theoretical point of view. As we are still lacking a bound state wave function for nucleon in terms of quarks and gluons, i.e. computed from first principles in QCD, it is both mandatory and fruitful to investigate these chiral odd flavor distributions and their charge weighted average nucleon structure functions within hadronic models of the nucleon [4, 5, 6, 7, 8, 9, 10].

In the context of the spin structure of the nucleon chiral soliton models are particularly interesting as they provide an explanation for the small magnitude of the quark spin contribution to the proton spin, i.e. the vanishingly small matrix element of the singlet axial current [11]. In these models the nucleon is described as a non-perturbative field configuration in some non-linear effective meson theory [12, 13, 14]. Unfortunately in many of these soliton models the evaluation of structure functions is infeasible due to the highly non-linear structure of the current operators and the inclusion of higher derivative operators which complicates the current commutation relations. However, it has recently been recognized that the soliton solution [14] which emerges after bosonization [15] of the Nambu-Jona-Lasinio (NJL) [16] chiral quark model can be employed to compute nucleon structure functions [17, 18]. In order to project this soliton configuration onto nucleon states with good spin and flavor a cranking procedure must be employed [13, 19] which implements significant $1 / N_{C}$ contributions $\left(N_{C}\right.$ is the number of color degrees of freedom.). When extracting the structure functions from the NJL chiral soliton model the full calculation which also includes effects of the vacuum polarized by the background

\footnotetext{
${ }^{1}$ The relevant fragmentation and distribution functions depend on different kinematical variables: the two meson state momentum fraction and the Bjorken variable, respectively.
} 
soliton is quite laborious. In addition we are still lacking a regularization prescription of the vacuum contribution to the structure functions which is derived from the action functional and which yields algebraic expressions for their moments which are consistent with those for the static nucleon properties. Fortunately it is known that the dominant contribution to static nucleon properties stems from the single quark level which has the lowest energy eigenvalue (in magnitude) and is strongly bound by the soliton [14. This is particularly the case for spin related quantities. Hence it is a reasonable approximation to consider only the contribution of this level to the structure functions. In the proceeding section the NJL chiral soliton model together with the above mentioned approximation, which we will call valence quark approximation 9 will be described in more detail.

The NJL model for the quark flavor dynamics incorporates spontaneous breaking of chiral symmetry in a dynamic fashion. Hence the quark fields which built up the soliton self-consistently [20] are constituent quarks with a constituent quark mass of several hundred MeV. Keeping this in mind we calculate both the effective constituent quark distributions and in turn the corresponding leading twist contributions to nucleon structure functions ( $c f$. eq (2)) at a low scale $Q_{0}^{2}$. In the language of Feynman diagrams the DIS processes are described by a constituent quark of the nucleon absorbing a quanta of the external source. In the Bjorken limit the quark then propagates highly off-shell before emitting a quanta of the external source. The intermediate quark may propagate forward and backward. Hence the complete structure functions acquire contributions from both distributions where the intermediate constituent quark moves forward and backward. We will focus on nucleon structure functions which are defined as the sum over the charge--weighted flavor distributions [4]

$$
h_{T / L}^{( \pm)}\left(x, Q_{0}^{2}\right)=\frac{1}{2} \sum_{a} e_{a}^{2} h_{T / L}^{(a, \pm)}\left(x, Q_{0}^{2}\right)
$$

in analogy to those of the chiral even spin polarized and unpolarized nucleon structure functions [2, 3]. Here a represents a quark label, while $( \pm)$ refers to the forward $(+)$ and backward (-) propagating intermediate constituent quarks. Furthermore $e_{a}$ denotes the charge fraction of the considered quark flavor $a$. The complete chiral odd structure functions are finally obtained as the sum

$$
h_{T / L}\left(x, Q_{0}^{2}\right)=h_{T / L}^{(+)}\left(x, Q_{0}^{2}\right)+h_{T / L}^{(-)}\left(x, Q_{0}^{2}\right)
$$

\footnotetext{
${ }^{2}$ This notation refers to the valence quark in the NJL chiral soliton model and should not be confused with the valence quark in the parton model.
} 
The calculation of the flavor distributions $h_{T / L}^{(a)}$ in the valence approximation to the NJL chiral soliton model [17, 18] is summarized in section 3 .

Further it is important to note that when considering model structure functions the OPE implies that the initial conditions, $\mu^{2}=Q_{0}^{2}$, for the evolution is, a priori, a free parameter in any baryon model [21]. For the model under consideration it has previously been determined to $Q_{0}^{2} \approx 0.4 \mathrm{GeV}^{2}$ by studying the evolution dependence of the model prediction for the unpolarized structure functions [17]. In a subsequent step to compute the chiral odd structure functions we employ a leading order evolution program [6, 9] to obtain the chiral odd structure functions at a larger scale, e.g. $Q^{2} \approx 4 \mathrm{GeV}^{2}$ relevant to the experimental conditions. This evolution program incorporates the leading logarithmic corrections to the leading twist pieces. The evolution procedure as applied to our model structure functions will be explained in section 4 .

The numerical results for the chiral odd structure functions are presented in section 5 while concluding remarks are contained in section 6 . Technical details on the model calculations and the QCD evolution procedure are relegated to appendices. Let us also mention that there has been a previous calculation of $h_{T}\left(x, Q_{0}^{2}\right)$ [10] which, however, ignored both the projection onto good nucleon states and the QCD evolution. Furthermore in that calculation an (arbitrary) meson profile was employed rather than a self-consistent soliton solution to the static equations of motion.

\section{The NJL-Model Chiral Soliton}

Before continuing with the discussion of the chiral odd structure functions, we will review the issue of the chiral soliton in the NJL model.

The Lagrangian of the NJL model in terms of quark degrees of freedom reads [16, 15]

$$
\mathcal{L}=\bar{q}\left(i \not \partial-m^{0}\right) q+2 G_{\mathrm{NJL}} \sum_{i=0}^{3}\left(\left(\bar{q} \frac{\tau^{i}}{2} q\right)^{2}+\left(\bar{q} \frac{\tau^{i}}{2} i \gamma_{5} q\right)^{2}\right)
$$

Here $q, \hat{m}^{0}$ and $G_{\text {NJL }}$ denote the quark field, the current quark mass and a dimensionful coupling constant, respectively. This model is motivated as follows: Integrating out the gluon fields from QCD yields a current-current interaction mediated by one gluon exchange to leading order in powers of the quark current. Replacing the gluon mediating propagator with a local contact interaction and performing the appropriate Fierz-transformations yields the Lagrangian (聿) in leading order of $1 / N_{C}$ [22, 23], where $N_{C}$ 
refers to the number of color degrees of freedom. Although only a subset of possible nonperturbative gluonic modes are contained in the contact interaction term in eq (田) it is important to stress that gluonic effects are contained in the model (4). Furthermore the NJL model embodies the approximate chiral symmetry of QCD and has to be understood as an effective (non-renormalizable) theory of the low-energy quark flavor dynamics.

Application of functional bosonization techniques [15] to the Lagrangian (4) yields the mesonic action

$$
\begin{aligned}
\mathcal{A} & =\operatorname{Tr}_{\Lambda} \log (D)+\frac{1}{4 G_{\mathrm{NJL}}} \int d^{4} x \operatorname{tr}\left(m^{0}\left(M+M^{\dagger}\right)-M M^{\dagger}\right), \\
D & =i \not \partial-\left(M+M^{\dagger}\right)-\gamma_{5}\left(M-M^{\dagger}\right),
\end{aligned}
$$

where $M=S+i P$ comprises composite scalar $(S)$ and pseudoscalar $(P)$ meson fields which appear as quark-antiquark bound states. For regularization, which is indicated by the cut-off $\Lambda$, we will adopt the proper-time scheme [24]. The free parameters of the model are the current quark mass $m^{0}$, the coupling constant $G_{\mathrm{NJL}}$ and the cut-off $\Lambda$. The equation of motion for the scalar field $S$ may be considered as the gap-equation for the order parameter $\langle\bar{q} q\rangle$ of chiral symmetry breaking. This equation relates the vacuum expectation value $\langle M\rangle=m \rrbracket$ to the model parameters $m^{0}, G_{\mathrm{NJL}}$ and $\Lambda$. For apparent reasons $m$ is called the constituent quark mass. The occurrence of this vacuum expectation value reflects the spontaneous breaking of chiral symmetry and causes the pseudoscalar fields to emerge as (would-be) Goldstone bosons. Expanding $\mathcal{A}$ to quadratic order in $P$ (around $\langle M\rangle$ ) these parameters are related to physical quantities; that is, the pion mass, $m_{\pi}=135 \mathrm{MeV}$ and the pion decay constant, $f_{\pi}=93 \mathrm{MeV}$. This leaves one undetermined parameter which we choose to be the constituent quark mass [15].

The NJL model chiral soliton [14, 20] is given by a non-perturbative meson configuration which is assumed of the hedgehog type

$$
M_{\mathrm{H}}(\boldsymbol{x})=m \exp (i \boldsymbol{\tau} \cdot \hat{\boldsymbol{x}} \Theta(r)) .
$$

In order to compute the functional trace in eq (5) for this static configuration we express the Dirac operator (6) in terms of a Hamiltonian operator $h$, i.e. $D=i \beta\left(\partial_{t}-h\right)$ with

$$
h=\boldsymbol{\alpha} \cdot \boldsymbol{p}+m \beta \exp \left(i \gamma_{5} \boldsymbol{\tau} \cdot \hat{\boldsymbol{x}} \Theta(r)\right)
$$

We denote the eigenvalues and eigenfunctions of $h$ by $\epsilon_{\mu}$ and $\Psi_{\mu}$, respectively. Explicit expressions for these wave-functions are displayed in appendix B of ref [14]. In the 
proper-time regularization scheme the energy functional of the NJL model is found to be [19, 14,

$$
\begin{aligned}
E[\Theta]=\frac{N_{C}}{2} \epsilon_{\mathrm{v}}\left(1+\operatorname{sgn}\left(\epsilon_{\mathrm{v}}\right)\right)+\frac{N_{C}}{2} \int_{1 / \Lambda^{2}}^{\infty} & \frac{d s}{\sqrt{4 \pi s^{3}}} \sum_{\nu} \exp \left(-s \epsilon_{\nu}^{2}\right) \\
& +m_{\pi}^{2} f_{\pi}^{2} \int d^{3} r(1-\cos \Theta(r)) .
\end{aligned}
$$

The subscript "v" denotes the valence quark level. This state is the distinct level bound in the soliton background, i.e. $-m<\epsilon_{\mathrm{v}}<m$. The chiral angle, $\Theta(r)$, is obtained by self-consistently extremizing $E[\Theta]$ [20].

States possessing nucleon quantum numbers of spin and isospin are generated by elevating the rotational zero modes to time dependent large amplitude rotational fluctuations about the hedgehog field [13]

$$
M(\boldsymbol{x}, t)=A(t) M_{\mathrm{H}}(\boldsymbol{x}) A^{\dagger}(t)
$$

which introduces the collective coordinates $A(t) \in S U(2)$. Substituting the ansatz (10) into the action functional (5) and expanding [19] in the angular velocities

$$
2 A^{\dagger}(t) \dot{A}(t)=i \boldsymbol{\tau} \cdot \Omega
$$

to quadratic order yields the Lagrange function for the collective coordinates. Upon canonical quantization the angular velocity $\Omega$ is substituted by the nucleon spin operator $\boldsymbol{J}=\alpha^{2} \boldsymbol{\Omega}$, with $\alpha^{2}$ being the moment of inertia [19, 14]. The eigenfunctions of the resulting Hamiltonian are the Wigner $D$-functions

$$
\langle A \mid N\rangle=\frac{1}{2 \pi} D_{I_{3},-J_{3}}^{1 / 2}(A),
$$

with $I_{3}$ and $J_{3}$ being respectively the isospin and spin projection quantum numbers of the nucleon. The nucleon matrix elements of the collective rotations are obtained via $\left\langle N\left|\operatorname{tr}\left(\tau_{i} A \tau_{j} A^{\dagger}\right)\right| N\right\rangle=-(8 / 3)\left\langle N\left|I_{i} J_{j}\right| N\right\rangle$ [13]. This approach to generate nucleon states from the hedgehog corresponds to the cranking technique in nuclear physics [25].

Expectation values of bilocal quark-bilinears appearing in the evaluation of nucleon structure functions are expressed as (regularized) sums over bilocal and bilinear combinations of all eigenfunctions $\Psi_{\mu}$ including the Dirac sea states. In practice this is quite a painful task, in particular when cranking corrections (10) are included. Also the problem of regularization is not consistently solved. Fortunately it turns out that the dominant contributions $(\geq 80 \%)$ to static nucleon properties (which are moments of the structure 
functions) stems from the distinct valence level $\Psi_{\mathrm{v}}$ [14. It is therefore reasonable to approximate the relevant bilinears by their valence quark contribution. In order to obtain quark distributions of the nucleon and the corresponding nucleon structure functions (c.f. 2), rather than soliton structure functions the cranking contribution to the wave-function, which is induced by the collective rotation $A(t)$, must be included. That is, the valence quark wave-function employed to approximate the bilinears in the structure functions reads

$$
\Psi_{\mathrm{v}}(\boldsymbol{x}, t)=\mathrm{e}^{-i \epsilon_{\mathrm{v}} t} A(t)\left\{\Psi_{\mathrm{v}}(\boldsymbol{x})+\frac{1}{2} \sum_{\mu \neq \mathrm{v}} \Psi_{\mu}(\boldsymbol{x}) \frac{\langle\mu|\boldsymbol{\tau} \cdot \Omega| \mathrm{v}\rangle}{\epsilon_{\mathrm{v}}-\epsilon_{\mu}}\right\}=: \mathrm{e}^{-i \epsilon_{\mathrm{v}} t} A(t) \psi_{\mathrm{v}}(\boldsymbol{x})
$$

Here $\psi_{\mathrm{v}}(\boldsymbol{x})$ refers to the spatial part of the body-fixed valence quark wave-function with the rotational corrections included and $\Psi_{\mu}=\langle\mu \mid \boldsymbol{x}\rangle$ are eigenfunctions of the Dirac Hamiltonian (8). This replacement of the bilocal and bilinear quark fields when computing nucleon structure functions defines the valence quark approximation.

\section{Chiral Odd Structure Functions, $h_{T}(x)$ and $h_{L}(x)$ in the NJL model}

Here we present the major topic of this paper, namely the calculation of the twist- 2 and twist-3 chiral odd structure functions in the NJL chiral soliton model. Like their deep inelastic chiral even (un)polarized counterparts, the chiral odd structure functions are computed as Fourier transformations of nucleon matrix elements of bilocal quark operators on the light-cone [4]. The key features of the relevant light-cone kinematics are given in Appendix A.

We begin by listing the forward propagating intermediate quark $(+)$ contribution to the chiral odd nucleon structure functions. Before, however, straightforwardly transcribing the expressions from appendix A we must recall that the soliton represents a localized field configuration. Therefore a collective coordinate $\boldsymbol{x}_{0}$ is introduced which parameterizes the position of the soliton. This collective coordinate is employed to generate states

with good linear momentum [26]. When computing matrix elements between states of identical momenta one is essentially left with an integration over $\boldsymbol{x}_{0}$. In the nucleon restframe $(\mathrm{RF})$ the contribution of the forward moving intermediate quark to the chiral odd 
structure functions may therefore be expressed as

$$
\begin{aligned}
h_{T}^{(+)}(x)=N_{C} \frac{2 M \sqrt{2}}{8 \pi} & \int d \xi^{-} \exp \left(-i \xi^{-} \frac{M x}{\sqrt{2}}\right) \\
& \times \int d^{3} \boldsymbol{x}_{0}\left\langle\boldsymbol{S}_{\perp}\left|\Psi_{+}^{\dagger}\left(\xi-\boldsymbol{x}_{0}\right) \gamma_{\perp} \gamma_{5} \mathcal{Q}^{2} \Psi_{+}\left(-\boldsymbol{x}_{0}\right)\right| \boldsymbol{S}_{\perp}\right\rangle_{\xi^{+}=\boldsymbol{\xi}_{\perp}=0} .
\end{aligned}
$$

For convenience we have omitted the subscript "v" for the valence quark wave function. Note that $\xi$ refers to a four vector which in light-cone coordinates reads $\left(\xi^{+}, \xi^{-}, \boldsymbol{\xi}_{\perp}\right)$. This coordinate enters the light-cone variables via $\xi^{ \pm}=(t \pm z) / \sqrt{2}$. Also, the notation $\boldsymbol{S}_{\perp}$ is synonymous for the spin being perpendicular to the coordinate $z$. On the other hand for the longitudinal counterpart

$$
\begin{aligned}
& h_{L}^{(+)}(x)=N_{C} \frac{2 M \sqrt{2}}{16 \pi} \int d \xi^{-} \exp \left(-i \xi^{-} \frac{M x}{\sqrt{2}}\right) \\
& \times \int d^{3} \boldsymbol{x}_{0}\left\langle\boldsymbol{S}_{z}\right| \Psi_{+}^{\dagger}\left(\xi-\boldsymbol{x}_{0}\right) \gamma_{0} \gamma_{5} \mathcal{Q}^{2} \Psi_{-}\left(-\boldsymbol{x}_{0}\right) \\
&-\Psi_{-}^{\dagger}\left(\xi-\boldsymbol{x}_{0}\right) \gamma_{0} \gamma_{5} \mathcal{Q}^{2} \Psi_{+}\left(-\boldsymbol{x}_{0}\right)\left|\boldsymbol{S}_{z}\right\rangle_{\xi^{+}=\boldsymbol{\xi}_{\perp}=0}
\end{aligned}
$$

the spin is aligned with the $z$-axis. The "good" and "bad" light-cone components of the quark wave functions are the projections $\Psi_{ \pm}=P_{ \pm} \Psi$, with $P_{ \pm}=\frac{1}{2} \gamma^{\mp} \gamma^{ \pm}$being the corresponding projections operators. Above, $\mathcal{Q}=\operatorname{diag}(2 / 3,-1 / 3)$ refers to the matrix containing the quark charge fractions and the zero momentum nucleon states are given by, $|\boldsymbol{p}=0, \boldsymbol{S}\rangle=\left[(2 \pi)^{3} 2 M\right]^{\frac{1}{2}}|\boldsymbol{S}\rangle$. Introducing Fourier transforms for the spatial part of the valence quark wave functions (cf. eq (13)),

$$
\psi\left(\boldsymbol{\xi}_{\perp}, \xi_{3}=-\frac{\xi^{-}}{\sqrt{2}}\right)=\int \frac{d^{2} p_{\perp} d p_{3}}{2 \pi^{2}} \exp \left[i\left(\frac{p_{3} \xi^{-}}{\sqrt{2}}-\boldsymbol{p}_{\perp} \cdot \boldsymbol{\xi}_{\perp}\right)\right] \tilde{\psi}\left(\boldsymbol{p}_{\perp}, p_{3}\right)
$$

yields,

$$
\begin{aligned}
h_{T}^{(+)}(x)=N_{C} \frac{M}{\sqrt{2} \pi^{2}} \int d \xi^{-} p^{2} d p d(\cos \theta) d \phi & \exp \left(\frac{-i \xi^{-}\left(M x-\epsilon_{\mathrm{v}}+p \cos \theta\right)}{\sqrt{2}}\right) \\
& \times\left\langle\boldsymbol{S}_{\perp}\left|\tilde{\psi}_{+}^{\dagger}(\boldsymbol{p}) \gamma_{\perp} \gamma_{5} \mathcal{Q}^{2} \tilde{\psi}_{+}(\boldsymbol{p})\right| \boldsymbol{S}_{\perp}\right\rangle
\end{aligned}
$$

and

$$
\begin{aligned}
h_{L}^{(+)}(x)=N_{C} & \frac{M}{\sqrt{2} \pi^{2}} \int d \xi^{-} p^{2} d p d(\cos \theta) d \phi \exp \left(\frac{-i \xi^{-}\left(M x-\epsilon_{\mathrm{v}}+p \cos \theta\right)}{\sqrt{2}}\right) \\
& \times\left\langle\boldsymbol{S}_{z}\left|\tilde{\psi}_{+}^{\dagger}(\boldsymbol{p}) \gamma_{0} \gamma_{5} \mathcal{Q}^{2} \tilde{\psi}_{-}(\boldsymbol{p})-\tilde{\psi}_{-}^{\dagger}(\boldsymbol{p}) \gamma_{0} \gamma_{5} \mathcal{Q}^{2} \tilde{\psi}_{+}(\boldsymbol{p})\right| \boldsymbol{S}_{z}\right\rangle .
\end{aligned}
$$

\footnotetext{
${ }^{3}$ The following expressions constitute a generalization of Jaffe's original definition [27] for nucleon structure functions.
} 
In anticipation of the decomposition (13) the square of the charge operator is redefined to

$$
\mathcal{Q}^{2}=\frac{5}{18} 1+\frac{1}{6} D_{3 i} \tau_{i}
$$

Here $D_{i j}=\frac{1}{2} \operatorname{tr}\left(\tau_{i} A(t) \tau_{j} A^{\dagger}\right)$ denotes the adjoint representation of the collective rotation which is defined in eq (10). The integrals over $\xi^{-}$and $\theta$ enforce both the constraint $\cos \theta=\left(\epsilon_{\mathrm{v}}-M x\right) / p$ and the lower bound, $p_{\min }=\left|M x-\epsilon_{\mathrm{v}}\right|$ on the $p$ integration. This results in the forward moving quark contributions to the transverse and longitudinal chiral odd nucleon structure functions

$$
h_{T}^{(+)}(x)=N_{C} \frac{2 M}{\pi} \int_{p_{\min }}^{\infty} p d p d \phi\left\langle\boldsymbol{S}_{\perp}\left|\tilde{\psi}_{+}^{\dagger}(\boldsymbol{p}) \gamma_{\perp} \gamma_{5} \mathcal{Q}^{2} \tilde{\psi}_{+}(\boldsymbol{p})\right| \boldsymbol{S}_{\perp}\right\rangle \mid \cos \theta=\frac{\epsilon-M x}{p}
$$

and

$$
\begin{aligned}
h_{L}^{(+)}(x)=N_{C} \frac{2 M}{\pi} \int_{p_{\min }}^{\infty} p d p & d \phi\left\langle\boldsymbol{S}_{z}\right| \tilde{\psi}_{+}^{\dagger}(\boldsymbol{p}) \gamma_{0} \gamma_{5} \mathcal{Q}^{2} \tilde{\psi}_{-}(\boldsymbol{p}) \\
& -\left.\tilde{\psi}_{-}^{\dagger}(\boldsymbol{p}) \gamma_{0} \gamma_{5} \mathcal{Q}^{2} \tilde{\psi}_{+}(\boldsymbol{p})\left|\boldsymbol{S}_{z}\right\rangle\right|_{\cos \theta=\frac{\epsilon-M x}{p}} .
\end{aligned}
$$

In order to obtain the full structure functions the contribution of backward moving quarks $h_{T, L}^{(-)}$must be considered as well. These contributions are easily obtained from $h_{T, L}^{(+)}(-x)$ by reversing the appropriate signs in equations (17) and (18). The two contributions may be comprised inf

$$
h_{T}^{( \pm)}(x)= \pm N_{C} \frac{M}{\pi} \int_{p_{\min }^{\mp}}^{\infty} p d p d \phi\left\langle\boldsymbol{S}_{\perp}\left|\tilde{\psi}^{\dagger}\left(\boldsymbol{p}_{\mp}\right)\left(1 \mp \alpha_{3}\right) \gamma_{\perp} \gamma_{5} \mathcal{Q}^{2} \tilde{\psi}\left(\boldsymbol{p}_{\mp}\right)\right| \boldsymbol{S}_{\perp}\right\rangle \mid \cos \theta_{p}^{\mp}
$$

and

$$
h_{L}^{( \pm)}(x)= \pm\left. N_{C} \frac{M}{\pi} \int_{p_{\min }^{\mp}}^{\infty} p d p d \phi\left\langle\boldsymbol{S}_{z}\left|\tilde{\psi}^{\dagger}\left(\boldsymbol{p}_{\mp}\right) \alpha_{3} \gamma_{0} \gamma_{5} \mathcal{Q}^{2} \tilde{\psi}\left(\boldsymbol{p}_{\mp}\right)\right| \boldsymbol{S}_{z}\right\rangle\right|_{\cos \theta_{p}^{\mp}}
$$

where $p_{\min }^{ \pm}=\left|M x \pm \epsilon_{\mathrm{v}}\right|$ and $\cos \theta^{ \pm}=\left(M x \pm \epsilon_{\mathrm{v}}\right) / p$ and $\tilde{\psi}\left(\boldsymbol{p}_{ \pm}\right)=\tilde{\psi}\left(p, \cos \theta_{p}^{ \pm}, \phi\right)$. Finally we summarize our results by decomposing the proton structure functions into their (iso)scalar and vector components,

$$
\begin{aligned}
& h_{T}(x)=h_{T,+}^{I=0}(x)+h_{T,+}^{I=1}(x)+\left(h_{T,-}^{I=0}(x)+h_{T,-}^{I=1}(x)\right) \\
& h_{L}(x)=h_{L,+}^{I=0}(x)+h_{L,+}^{I=1}(x)+\left(h_{L,-}^{I=0}(x)+h_{L,-}^{I=1}(x)\right) .
\end{aligned}
$$

The isoscalar piece $(I=0)$ originates from the unit matrix in the decomposition (19) while the isovector part $(I=1)$ stems from the terms involving the collective coordinates. The explicit expressions for the structure functions (24) in terms of the static quark wave functions are computed in Appendix B.

\footnotetext{
${ }^{4}$ We have used that the valence quark level has positive parity, i.e. under $\boldsymbol{p} \rightarrow-\boldsymbol{p}$ we find $\tilde{\psi}(-\boldsymbol{p})=$ $\gamma^{0} \tilde{\psi}(\boldsymbol{p})$.
} 


\section{Projection And Evolution}

We consider that our model approximates QCD at a low scale $Q_{0}^{2}$. In oder to compare the predicted structure functions with data they must be evolved to a (larger) $Q^{2}$ commensurate with experimental conditions. A direct comparison with data gathered at a low scale cannot be made as the latter structure functions contain sizable contributions from higher twist. Thus we evolve the chiral odd model structure functions of the preceding section utilizing the results of perturbative QCD.

In the soliton approach the baryon states are built from localized field configurations. In fact, these states do not carry good four-momentum. Therefore the calculated structure functions ( $c f$. Figs. 1, 国 and 4) do not vanish exactly for $x>1$ although the contributions for $x>1$ are very small.
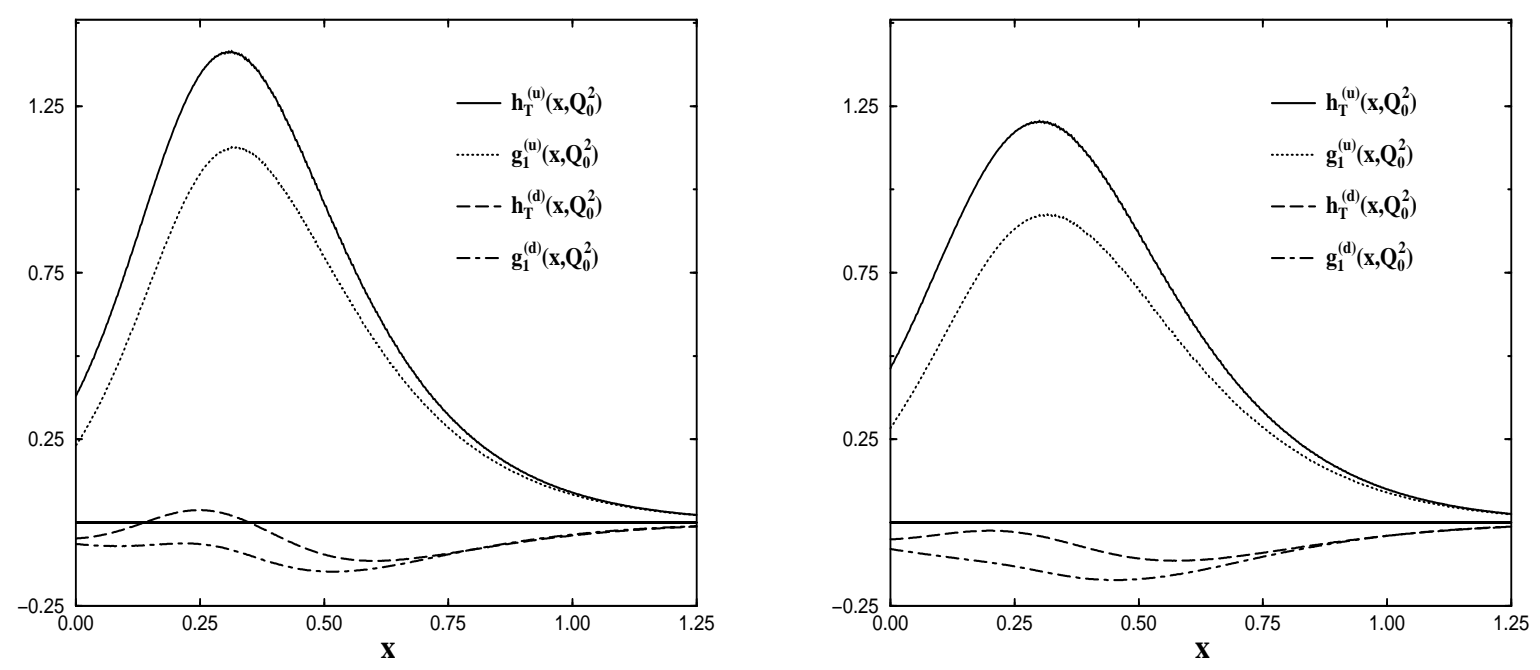

Figure 1: The valence quark approximation of the transverse chiral-odd nucleon distribution function as a function of Bjorken- $x$ for the up and down quark flavor content in the rest frame. For comparison also the model calculation [18] for the twist-2 polarized structure function $g_{1}\left(x, Q_{0}^{2}\right)$ is shown for the respective flavor channels. Two values of the constituent quark mass are considered: $m=400 \mathrm{MeV}$ (left panel) and $m=450 \mathrm{MeV}$ (right panel).

The calculation of nucleon structure functions in the Bjorken limit, however, singles out the null plane, $\xi^{+}=0$. This condition can be satisfied upon transformation to the infinite momentum frame (IMF) even for models where the nucleon emerges as a (static) localized object [28]. For the quark soliton model under consideration this transformation 
corresponds to performing a boost in the space of the collective coordinate $\boldsymbol{x}_{0}, c f$. eq (14). Upon this boost to the IMF we have observed [29] that the common problem of improper support for the structure functions, i.e. non-vanishing structure functions for $x>1$, is cured along the line suggested by Jaffe [30] some time ago. The reason simply is that the Lorentz contraction associated with the boost to the IMF maps the infinite line exactly onto the interval $x \in[0,1[$. In addition we have observed that this Lorentz contraction effects the structure functions also at small and moderate $x$. Incorporating these results for the general set of leading twist structure functions within the NJL-chiral soliton model yields the following form for the forward and backward moving intermediate quark state contributions to the chiral odd transverse spin structure function, $h_{T}^{( \pm)}\left(x, Q^{2}\right)$,

$$
\begin{aligned}
h_{T}^{( \pm)}(x)= \pm N_{C} & \frac{M}{\pi(1-x)} \int_{p_{\min }}^{\infty} p d p d \varphi \\
& \times\left.\left\langle N\left|\tilde{\psi}^{\dagger}\left(\boldsymbol{p}_{\mp}\right)\left(1 \mp \alpha_{3}\right) \gamma_{\perp} \gamma_{5} \mathcal{Q}^{2} \tilde{\psi}\left(\boldsymbol{p}_{\mp}\right)\right| N\right\rangle\right|_{\cos \theta=-\frac{M \ln (1-x) \pm \epsilon_{\mathrm{v}}}{p}} .
\end{aligned}
$$

In general the resulting relation between structure functions in the IMF and the rest frame $(\mathrm{RF})$ reads

$$
f_{\mathrm{IMF}}(x)=\frac{\Theta(1-x)}{1-x} f_{\mathrm{RF}}(-\ln (1-x))
$$

Of course, in the context of the chiral odd structure functions $f_{\mathrm{RF}}$ is to be identified with the expressions in eqs $(22,23,24)$. As will be recognized shortly the solution to the proper support problem is essential in order to apply the evolution program of perturbative QCD. The chiral odd and polarized structure functions resulting from this transformation are shown in figure 2 .

In order to include the logarithmic corrections to the twist-2 pieces of the chiral odd structure functions we apply the well-established GLAP procedure [31]. For the transverse component $h_{T}\left(x, Q^{2}\right)$ this is straightforward as it is pure twist-2. For the longitudinal piece $h_{L}\left(x, Q^{2}\right)$ one first has to extract the twist-2 component through $h_{T}\left(x, Q^{2}\right)$ namely, $h_{L}^{(2)}\left(x, Q^{2}\right)=2 x \int_{x}^{1} d y h_{T}\left(y, Q^{2}\right) / y^{2}$.

We simultaneously denote by $h^{(2)}$ the twist-2 parts of $h_{T}$ and $h_{L}$. To leading order (in $\left.\alpha_{Q C D}\left(Q^{2}\right)\right)$ the variations of the structure functions from a change $\delta t$ of the momentum scale is given by

$$
h^{(2)}(x, t+\delta t)=h^{(2)}(x, t)+\frac{d h^{(2)}(x, t)}{d t} \delta t,
$$

where $t=\log \left(Q^{2} / \Lambda_{Q C D}^{2}\right)$. The variation (27) is essentially due to the emission and absorption of soft gluons. The explicit expression for the evolution differential equation 

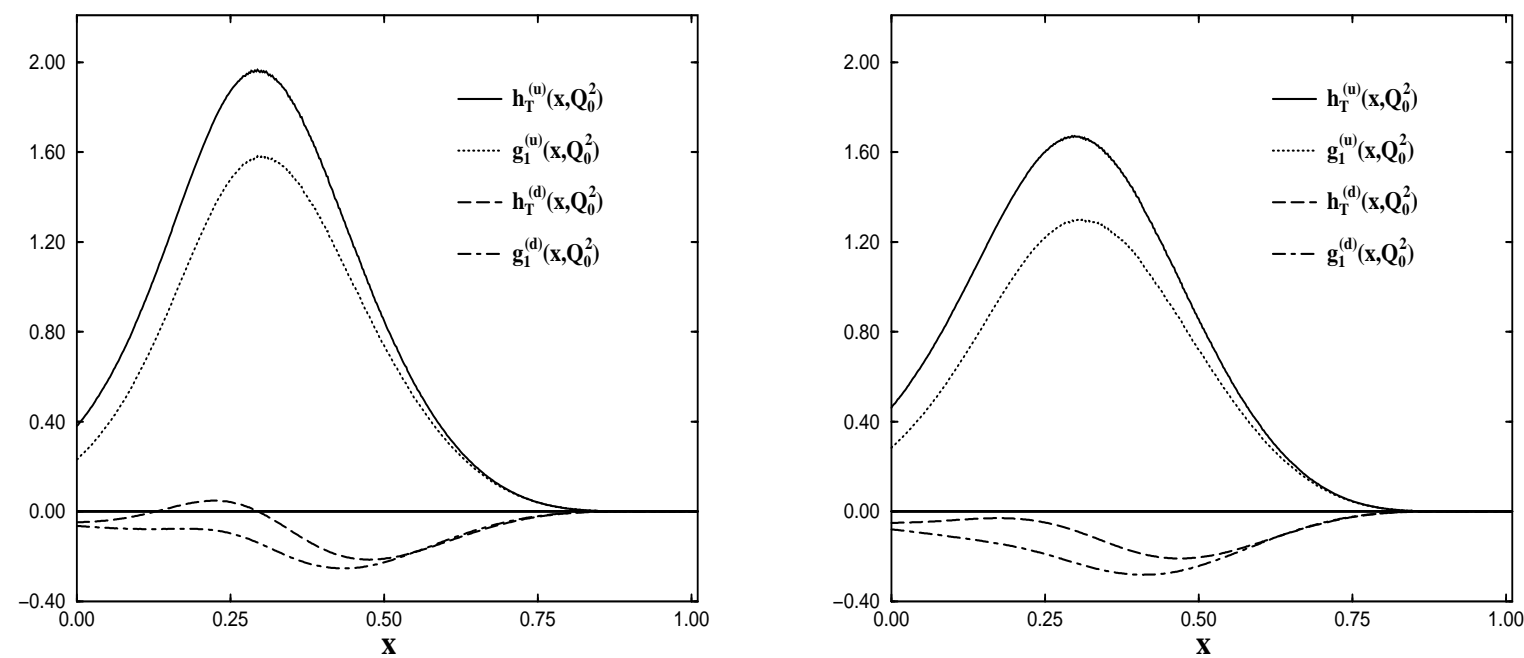

Figure 2: Same as figure 1 in the IMF (26).

is given by the convolution integral,

$$
\frac{d h^{(2)}(x, t)}{d t}=\frac{\alpha_{Q C D}(t)}{2 \pi} C_{R}(F) \int_{x}^{1} \frac{d y}{y} P_{q q}^{h}(y) h^{(2)}\left(\frac{x}{y}, t\right)
$$

where the leading order splitting function [32, 6] is given by,

$$
P_{q q}^{h}(z)=\frac{4}{3}\left[\frac{2}{(1-z)_{+}}-2+\frac{3}{2} \delta(z-1)\right]
$$

and $C_{R}(f)=\left(n_{f}^{2}-1\right) / 2 n_{f}$ for $n_{f}$ active flavors, $\alpha_{Q C D}(t)=4 \pi /\left[b_{0} \log \left(Q^{2} / \Lambda^{2}\right)\right]$ and $b_{0}=\left(11 N_{C}-2 n_{f}\right) / 3$. Employing the "+" prescription yields for three light flavors and $N_{C}=3$

$$
\begin{aligned}
\frac{d h^{(2)}(x, t)}{d t} & =\frac{\alpha_{Q C D}(t)}{2 \pi}\left\{\left(2+\frac{8}{3} \log (1-x)\right) h^{(2)}(x, t)\right. \\
& \left.+\frac{8}{3} \int_{x}^{1} \frac{d y}{y}\left[\frac{1}{1-y}\left(h^{(2)}\left(\frac{x}{y}, t\right)-y h^{(2)}(x, t)\right)-h^{(2)}\left(\frac{x}{y}, t\right)\right]\right\}
\end{aligned}
$$

As indicated above, the structure functions must vanish at the boundary $x=1$ in order to cancel the divergence of the logarithm in eq (30) and thus for the GLAP procedure to be applicable. This makes the projection of the rest frame structure functions mandatory. The variation of the structure functions for finite intervals in $t$ is straightforwardly obtained by iteration of these equations, i.e. as a solution to the differential equation (30). As discussed previously the initial value for integrating the differential equation is given 
by the scale $Q_{0}^{2}$ at which the model is defined. It should be emphasized that this scale essentially is a new parameter of the model. For a given constituent quark mass $m$ we adjust $Q_{0}^{2}$ to maximize the agreement of the predictions with the experimental data on previously [17] calculated unpolarized structure functions for electron-nucleon DIS: $F_{2}^{e p}-F_{2}^{e n}$. For the constituent quark mass $m=400 \mathrm{MeV}$ we have obtained $Q_{0}^{2} \approx 0.4 \mathrm{GeV}^{2}$. Note that this value of $Q_{0}^{2}$ is indeed (as it should) smaller than the ultraviolet cut-off of the underlying NJL soliton model as $\Lambda^{2} \approx 0.56 \mathrm{GeV}^{2}$. The latter quantity indicates the range of validity of the model. In figure 5a we compare the un-evolved, projected, proton structure function $h_{T}^{p}\left(x, Q_{0}^{2}\right)$ with the one evolved from $Q_{0}^{2}=0.4 \mathrm{GeV}^{2}$ to $Q^{2}=4.0 \mathrm{GeV}^{2}$. As expected the evolution pronounces the structure function at low $x$.

This change towards small $x$ is a generic feature of the projection and evolution process and presumably not very sensitive to the prescription applied here. In particular, choosing a projection technique [33] alternative to (26) may easily be compensated by an appropriate variation of the scale $Q_{0}^{2}$. In figure $5 \mathrm{~b}$ the same calculation for $h_{L}^{(2)}\left(x, Q^{2}\right)$ is presented.

In the evolution of the twist-2 pieces we have restricted ourselves to the leading order in $\alpha_{s}$ because for the twist-3 piece of $h_{L}$, the necessary ingredients are not known in next-to-leading order. Even the leading order evolution is only known in the large $N_{C}$ limit. It should be noted that such an approach seems particularly suited for soliton models which equally utilize large $N_{C}$ arguments. As pointed out by Balitskii et al. [34 the admixture of independent quark and quark-gluon operators contributing to the twist-3 portion $\bar{h}_{L}\left(x, Q^{2}\right)$ grows with $n$ where $n$ refers to the $n^{\text {th }}$ moment, $\mathcal{M}_{n}\left[\bar{h}_{L}\left(Q^{2}\right)\right]$ of $h_{L}\left(x, Q^{2}\right)$. However, much like the case with the spin-polarized structure function, $g_{2}\left(x, Q^{2}\right)$ 35 in the $N_{C} \rightarrow \infty$ limit the quark operators of twist-3 decouple from the quark-gluon operators of the same twist. Then the anomalous dimensions $\gamma_{n}$ which govern the logarithmic $Q^{2}$ dependence of $\mathcal{M}_{n}$ can be computed. Once the $\gamma_{n}$ 's are known an evolution kernel can be constructed that "propagates" the the twist-3 part $\bar{h}\left(x, Q^{2}\right)$ in momentum

$$
\bar{h}_{L}\left(x, Q^{2}\right)=\int_{x}^{1} \frac{d y}{y} b\left(x, y ; Q^{2}, Q_{0}^{2}\right) \bar{h}_{L}\left(y, Q_{0}^{2}\right)
$$

We relegate the detailed discussion of the kernel $b\left(x, y ; Q^{2}, Q_{0}^{2}\right)$, which is obtained by inverting the $Q^{2}$ dependence of $\mathcal{M}_{n}$, to appendix C. In figure 6 a we show the evolution of $\bar{h}_{L}(x)$. Again we used $Q_{0}^{2}=0.4 \mathrm{GeV}^{2}$ and $Q^{2}=4.0 \mathrm{GeV}^{2}$.

As discussed in ref [34] the merit of this approach is that to leading order in $N_{C}$ 
the knowledge of $h_{L}\left(x, Q^{2}\right)$ at one scale is sufficient to predict it at any arbitrary scale, which is not the case at finite $N_{C} \cdot{ }^{5}$ Thus $h_{L}\left(x, Q^{2}\right)$ obeys a generalized GLAP evolution equation. This finally enables us (in much the same manner as was the case for $g_{2}\left(x, Q^{2}\right)$ in [18]) to compute the longitudinal chiral odd structure function $h_{L}\left(x, Q^{2}\right)$ by combining the separately evolved twist- 2 and twist -3 components together. The result for $Q_{0}^{2}=0.4 \mathrm{GeV}^{2}$ and $Q^{2}=4.0 \mathrm{GeV}^{2}$ is shown in figure $6 \mathrm{~b}$. We recall that the only ingredients have been the leading twist pieces of the chiral odd structure functions at the model scale $Q_{0} \cdot$ f $^{\prime}$

\section{Discussion of the Numerical Results}

In this section we discuss the results of the chiral-odd structure functions calculated from eqs (59)-(62) for constituent quark masses $m=400 \mathrm{MeV}$ and $m=450 \mathrm{MeV}$. In figure 1 we have shown the up and down quark contributions to the transverse chiral odd structure function of the proton. Figure 2 displays them boosted to the IMF. We observe that these structure functions are always smaller (in magnitude) than the twist-2 polarized structure function $g_{1}$ with the same flavor content. This relation is also known from the bag model [4]. Similar to the confinement model calculation of Barone et al. [6] we find that $h_{T}^{(d)}(x)$ is negative at small $x$. In contrast to $g_{1}^{(d)}(x)$, however, it might change sign although the positive contribution appears to be small and diminishing with increasing constituent quark mass.

As already indicated in the introduction the DIS processes which are sensitive to these distributions will provide access to the charge weighted combinations thereof. We will hence concentrate on this flavor content. In any event, as we will be discussing both, the proton and the neutron chiral odd distributions, other flavor combinations can straightforwardly be extracted by disentangling the isoscalar and isovector pieces in eq (19). In connection with the chiral-odd transverse nucleon structure function we also calculate its zeroth moment which is referred to as the isoscalar and isovector nucleon tensor charges [4],

$$
\Gamma_{T}^{S}\left(Q^{2}\right)=\frac{18}{5} \int_{0}^{1}\left[d x h_{T}^{p}\left(x, Q^{2}\right)+h_{T}^{n}\left(x, Q^{2}\right)\right]
$$

\footnotetext{
${ }^{5}$ As noted in [34], next to leading order corrections are estimated to go like $O\left(1 / N_{c}^{2} \times \ln (n) / n\right)$ at large $n$.

${ }^{6} \mathrm{~A}$ feature of $h_{L}(x)$ compared with $g_{2}(x)$ is that as $h_{L}(x)$ does not mix with gluon distributions owing to its chiral-odd nature and its $Q^{2}$ evolution is given by (65), (66) even for the flavor singlet piece.
} 


$$
\Gamma_{T}^{V}\left(Q^{2}\right)=6 \int_{0}^{1}\left[d x h_{T}^{p}\left(x, Q^{2}\right)-h_{T}^{n}\left(x, Q^{2}\right)\right]
$$

at both the low scale, $Q_{0}^{2}=0.4 \mathrm{GeV}^{2}$ and a scale commensurate with experiment, $Q^{2}=$ $4 \mathrm{GeV}^{2}$. Of course, for the neutron we have to reverse the signs of the isovector pieces in eq (24). In eqs (32) and (33) the normalization factors are due to the separation into isosinglet and isovector contributions, $c f$. eq (19). Note that due to $\int_{0}^{1} d z P_{q q}^{h}(z) \neq 0$ the tensor charge is not protected against logarithmic corrections. Our results for the valence quark approximation are summarized in Table 1. For completeness we also add the vacuum contribution to the tensor charges at the model scale $Q_{0}^{2}$. Their analytic expressions are given in appendix D. Obviously this vacuum contribution is negligibly small. This is a strong justification of the valence quark approximation to the chiral odd structure functions. A further justification comes from a recent study of the Gottfried

Table 1: Nucleon tensor charges calculated from eqs (32) and (33) as a function of the constituent quark mass $m$ in the NJL chiral-soliton model. The momentum scales are $Q_{0}^{2}=0.4 \mathrm{GeV}^{2}$ and $Q^{2}=4.0 \mathrm{GeV}^{2}$. The numbers in parenthesis in the respective upper rows include the negligible contribution from the polarized quark vacuum. We compare with results from the Lattice [36], QCD sum rules [37], the constituent quark model with Goldstone boson effects [38] and a quark soliton model calculation 39] including multiplicative $1 / N_{C}$ corrections violating PCAC in the similar case of the axial vector current [40]. Finally the predictions from the confinement model of ref [6] with the associated momentum scales (in $\mathrm{GeV}^{2}$ ) are shown.

\begin{tabular}{c|lll|rrrr|ccc}
$m(\mathrm{MeV})$ & \multicolumn{1}{|c|}{350} & 400 & 450 & Lat. & SR & CQ & QS & $Q^{2}$ & CM \\
\hline$\Gamma_{T}^{S}\left(Q_{0}^{2}\right)$ & $0.80(0.82)$ & $0.72(0.76)$ & $0.67(0.72)$ & 0.61 & 0.61 & 1.31 & 0.69 & 0.16 & 0.90 \\
$\Gamma_{T}^{S}\left(Q^{2}\right)$ & 0.73 & 0.65 & 0.61 & no scale attributed & 25.0 & 0.72 \\
\hline$\Gamma_{T}^{V}\left(Q_{0}^{2}\right)$ & $0.88(0.89)$ & $0.86(0.87)$ & $0.86(0.85)$ & 1.07 & 1.37 & 1.07 & 1.45 & 0.16 & 1.53 \\
$\Gamma_{T}^{V}\left(Q^{2}\right)$ & 0.80 & 0.78 & 0.77 & \multicolumn{2}{|c|}{ no scale attributed } & 25.0 & 1.22
\end{tabular}

sum rule within the same model [41]. Also in that case the contribution of the distorted quark vacuum to the relevant structure function turned out to be negligibly small.

Besides justifying the valence quark approximation for the chiral odd distributions table 1 contains the comparison to other model calculations of the nucleon tensor charges. We note that in obtaining the isovector tensor charge $\Gamma_{T}^{V}$ we have omitted contributions which are suppressed by $1 / N_{C}(c f$. appendix D). These contributions arise when one adopts a non-symmetric ordering of the operators in the space of the collective operators [39]. The main reason for taking the symmetric ordering is that in the case of the isovector 
axial charge, $g_{A}$, any non-symmetric ordering of the collective operators leads to a sizable violation of PCAC unless the meson profile is not modified 40. These multiplicative $1 / N_{C}$ corrections [42] may be the reason why our predictions for $\Gamma_{T}^{V}$ are somewhat lower than those of other models. In the case of the flavor singlet component, which does not have such corrections, our results compare nicely with other model calculations except for the constituent quark model of ref [38].

In figure 3 we display the transverse chiral odd proton $h_{T}^{p}\left(x, Q_{0}^{2}\right)$ and neutron $h_{T}^{n}\left(x, Q_{0}^{2}\right)$ structure functions at the low momentum scale $Q_{0}^{2}$, while in figure 6 we do the same for the corresponding chiral odd longitudinal structure functions $h_{L}^{p}\left(x, Q_{0}^{2}\right)$ and $h_{L}^{n}\left(x, Q_{0}^{2}\right)$.
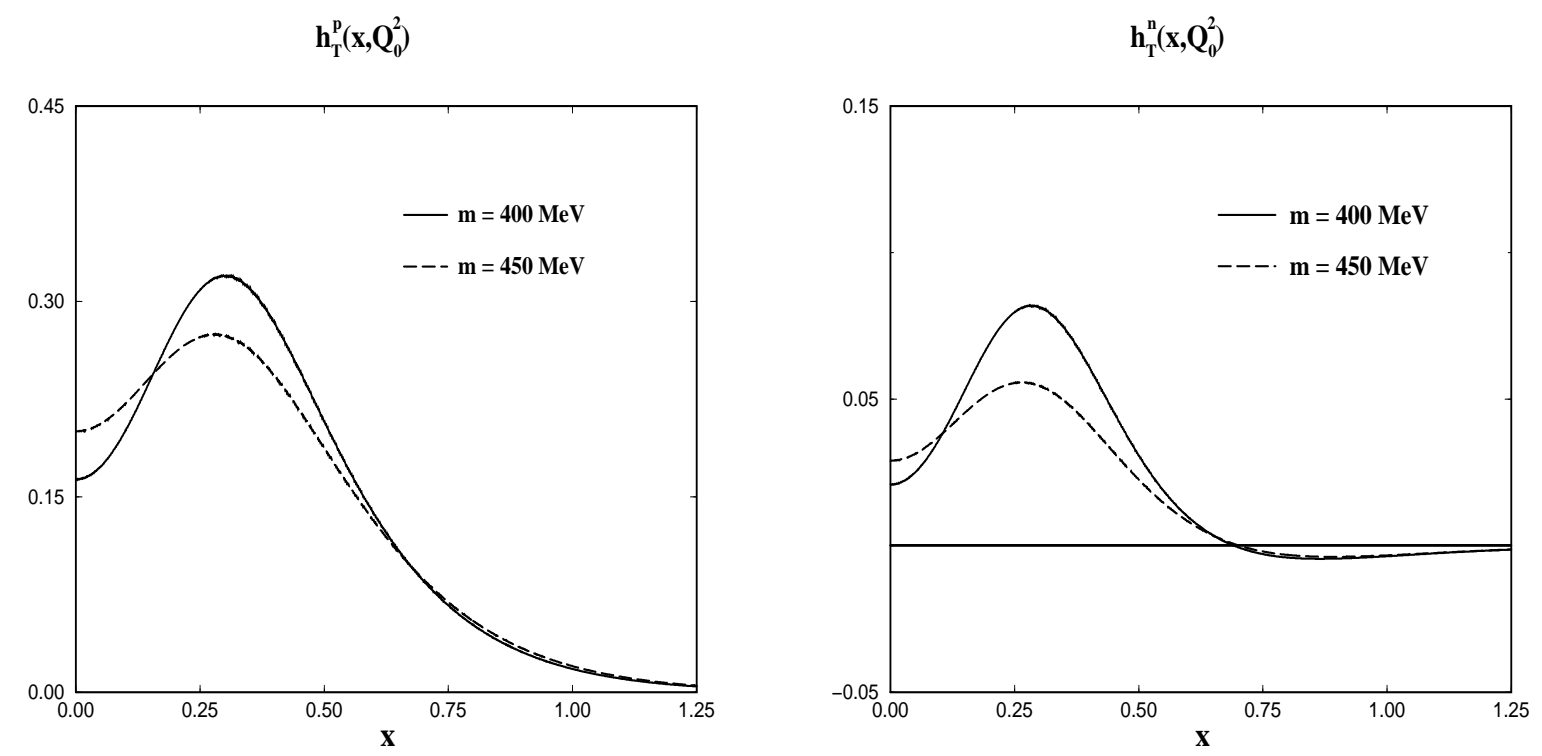

Figure 3: The valence quark approximation of the chiral-odd nucleon structure functions as a function of Bjorken- $x$. Left panel: $h_{T}^{p}\left(x, Q_{0}^{2}\right)$ for constituent quark masses $m=$ $400 \mathrm{MeV}$ (solid line) and $m=450 \mathrm{MeV}$ (long-dashed line). Right panel: $h_{T}^{n}\left(x, Q_{0}^{2}\right)$.

We observe that the structure functions $h_{T}^{N}\left(x, Q_{0}^{2}\right)$ and $h_{L}^{N}\left(x, Q_{0}^{2}\right)$ are reasonably localized in the interval $0 \leq x \leq 1$. In particular, this is the case for the chiral odd structure functions of the neutron. Nevertheless a projection as in eq (26) is required to implement Lorentz covariance. In addition the computed structure functions exhibit a pronounced maximum at $x \approx 0.3$ which is smeared out when the constituent quark mass $m$ increases. This can be understood as follows: In our chiral soliton model the constituent mass serves as a coupling constant of the quarks to the chiral field (see eqs (5) and (8)). The valence quark level becomes more strongly bound as the constituent quark mass increases. Hence the lower components of the valence quark wave-function increase with $m$ and relativistic effects become more important. This effect results in the above mentioned broadening of 

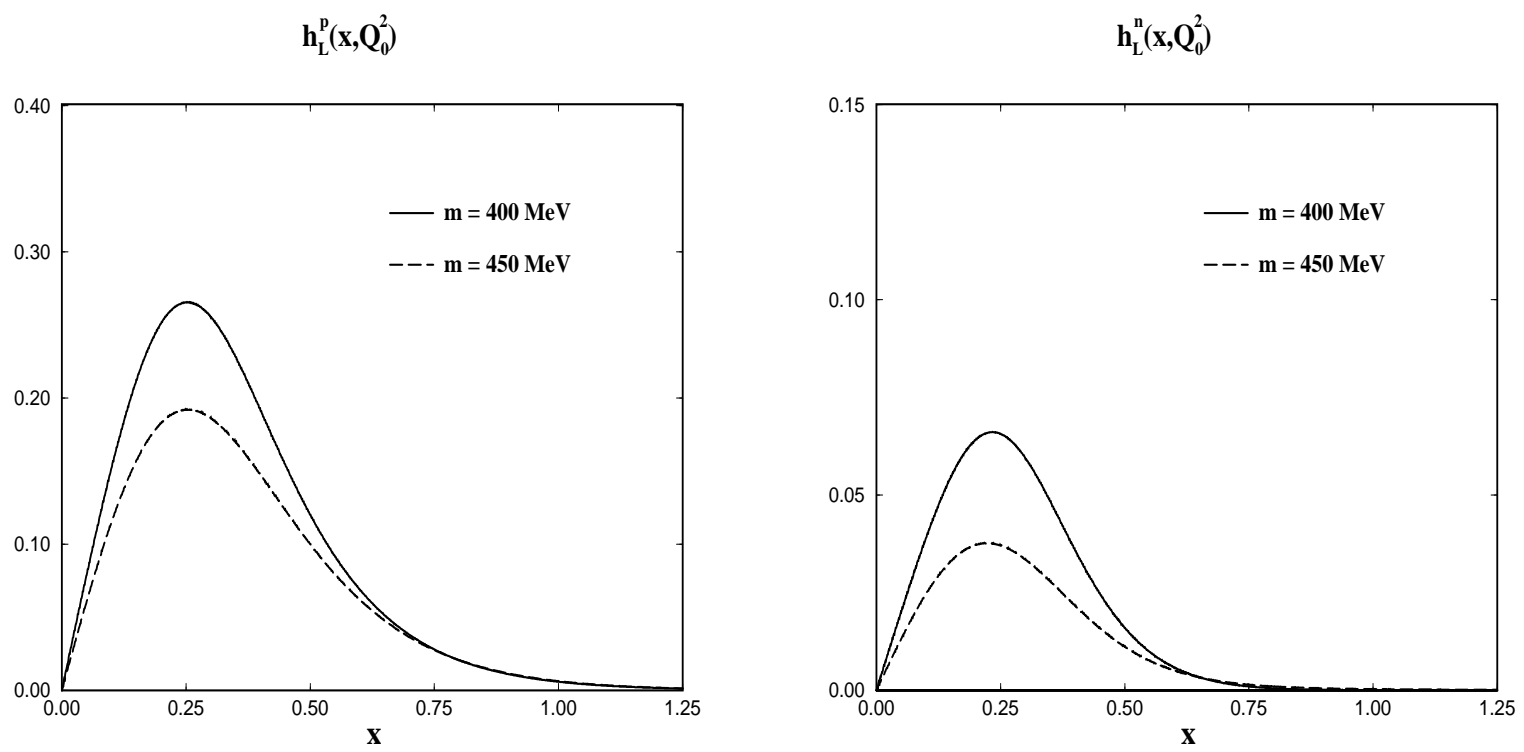

Figure 4: The valence quark approximation of the chiral-odd nucleon structure functions as a function of Bjorken- $x$. Left panel: $h_{L}^{p}\left(x, Q_{0}^{2}\right)$ for constituent quark masses $m=$ $400 \mathrm{MeV}$ (solid line) and $m=450 \mathrm{MeV}$ (long-dashed line). Right panel: $h_{L}^{n}\left(x, Q_{0}^{2}\right)$.

the maximum.

As discussed above a sensible comparison with (eventually available) data requires either to evolve the model results upward according to the QCD renormalization group equations or to compare the model results with a low momentum scale parameterization of the leading twist pieces of the structure functions. The latter requires the knowledge of the structure functions at some scale in the whole interval $x \in[0,1[$. At present no such data are available for the chiral odd structure functions $h_{T}(x)$ and $h_{L}(x)$. Therefore and in anticipation of results from RHIC and or HERMES we apply leading order evolution procedures to evolve the structure function from the model scale, $Q_{0}^{2}=0.4 \mathrm{GeV}^{2}$ to $Q^{2}=4 \mathrm{GeV}^{2}$. In Figs. $5 \mathrm{a}$ and $5 \mathrm{~b}$ we display the results of the two step process of projection and evolution for the twist-2 transverse structure function, $h_{T}^{p}\left(x, Q^{2}\right)$ and $h_{L}^{p(2)}\left(x, Q^{2}\right)$, respectively for a constituent quark mass of $m=400 \mathrm{MeV}$. In figure 6 we present the evolution of $h_{L}^{p}(x)$ along with its decomposition into terms of the leading twist-2 contribution, $2 x \int_{x}^{1} d y h_{T}^{p}\left(y, Q^{2}\right) / y^{2}$, and the remaining twist-3 piece, $\bar{h}_{L}^{p}\left(x, Q^{2}\right)$. As in the case of the polarized structure function, $g_{2}\left(x, Q^{2}\right)$, the non-trivial twist-3 piece arises as a result of the binding of the constituent quarks through the pion fields acting as effective non-perturbative gluonic modes. The twist- 3 contribution is evolved according to the large $N_{C}$ scheme [34, 35, 43] outlined in the preceding section (and in Appendix C). Similarly in Figs. 7 and 8 we display the projection and evolution procedure to the 

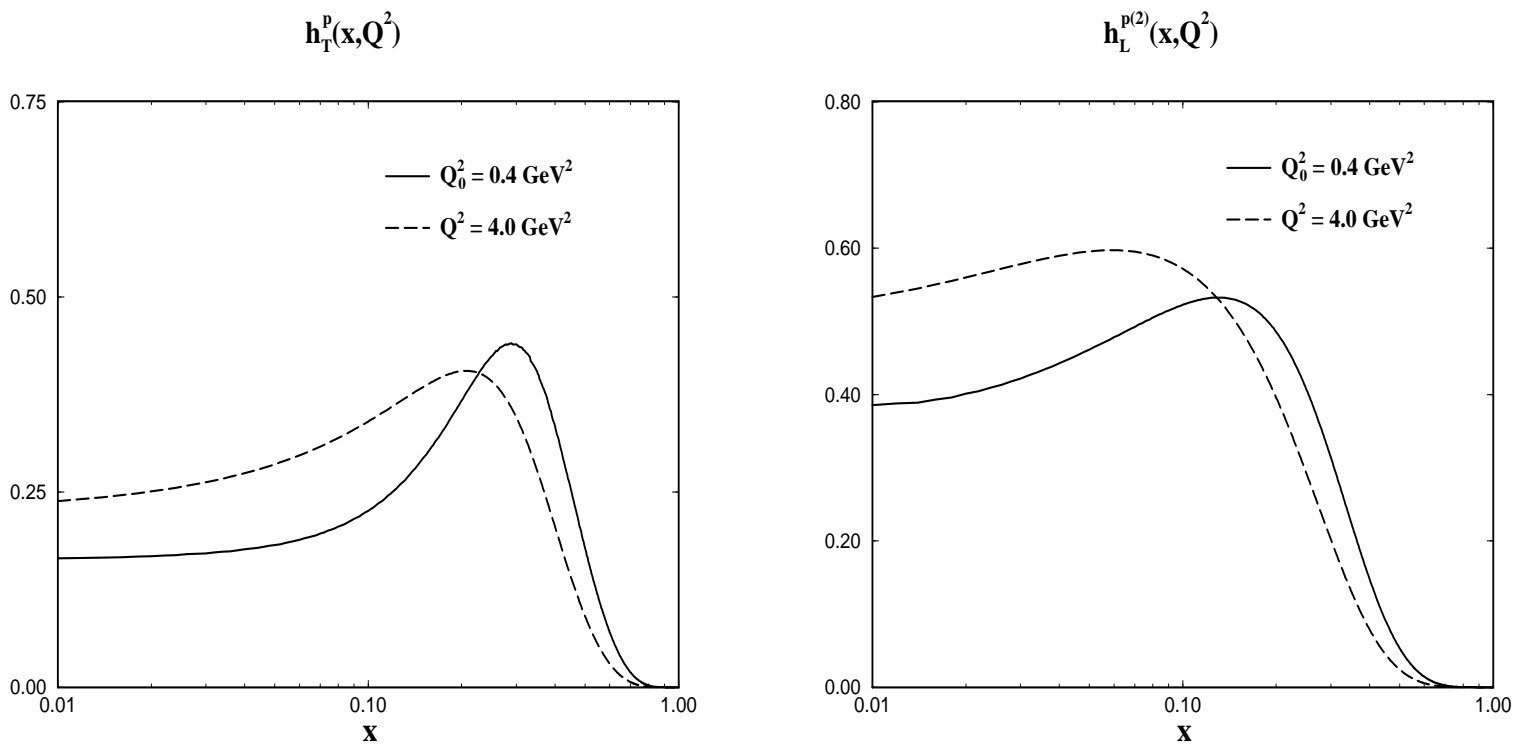

Figure 5: Left panel: The evolution of $h_{T}^{p}\left(x, Q^{2}\right)$ from $Q_{0}^{2}=0.4 \mathrm{GeV}^{2}$ (solid line) to $Q^{2}=4 \mathrm{GeV}^{2}$ (long-dashed line) for the constituent quark mass $m=400 \mathrm{MeV}$. Right panel: The evolution of the twist-2 contribution to the longitudinal chiral odd structure function, $h_{L}^{p(2)}\left(x, Q^{2}\right)$ from $Q_{0}^{2}=0.4 \mathrm{GeV}^{2}$ (solid line) to $Q^{2}=4 \mathrm{GeV}^{2}$ (long-dashed line) for $m=400 \mathrm{MeV}$.

twist-2 and 3 contribution to the neutron structure functions, $h_{L}^{n(2)}\left(x, Q^{2}\right)$ and $\bar{h}_{L}^{n}\left(x, Q^{2}\right)$, respectively.

Besides the absolute magnitudes, the major difference between the chiral odd structure functions of the proton and the neutron is that the latter drop to zero at a lower value of $x$. As can be observed from figure 3 this is inherited from the model chiral odd structure function at the low momentum scale and can be linked to the smallness of the down quark component of $h_{T}$, cf. figure [1. Apparently the projection and evolution program does not alter this picture.

We would also like to compare our results from the NJL chiral soliton model to those obtained in other approaches. A MIT bag model calculation of the isovector contribution $6\left(h_{T}^{p}-h_{T}^{n}\right)$ has been presented in ref [4]. In shape (e.g. position of the maximum) that result is quite similar to ours. However, the absolute value is a bit larger in the MIT bag model. This reflects the fact that in the MIT bag model the isovector combinations of the axial and tensor charges turn out to be bigger than in the present model. Additionally, the QCD evolution of the MIT bag model prediction for $h_{T}$ has been studied in ref [5] utilizing the Peierls-Yoccoz projection as in ref [21]. In that case the maximum at $x \approx 0.5$ gets shifted to a value as low as $x=0.2$. Also the structure function becomes rather broad at 

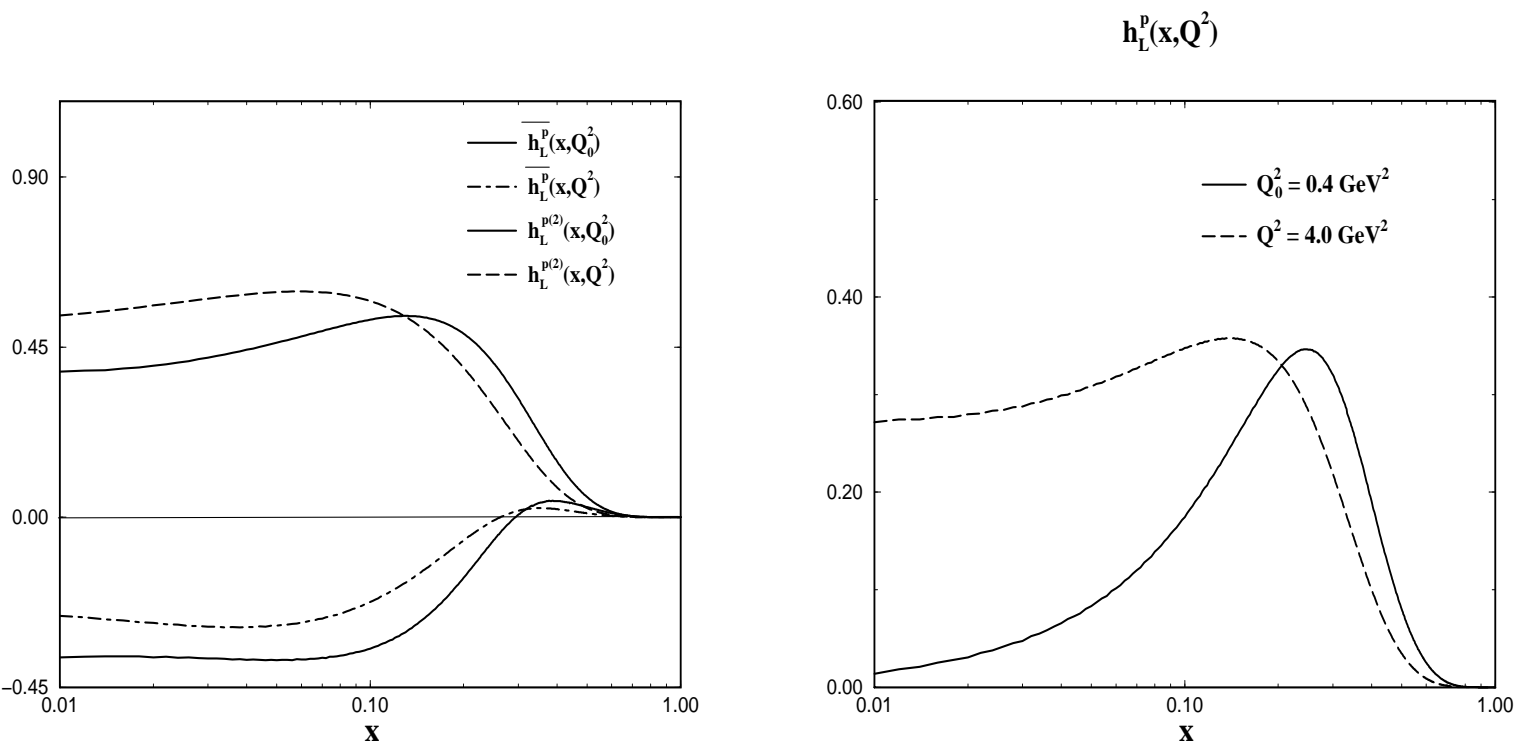

Figure 6: Left panel (6a): The evolution of the twist-3 contribution to the longitudinal chiral odd structure function, $\bar{h}_{L}^{p}\left(x, Q^{2}\right)$ along with the corresponding twist-2 piece, $h_{L}^{p(2)}\left(x, Q^{2}\right)$. Right panel (66) : The evolution of $h_{L}^{p}\left(x, Q^{2}\right)=h_{L}^{p(2)}\left(x, Q^{2}\right)+\bar{h}_{L}^{p}\left(x, Q^{2}\right)$ from $Q_{0}^{2}=0.4 \mathrm{GeV}^{2}$ (solid line) to $Q^{2}=4 \mathrm{GeV}^{2}$ (long-dashed line) for the constituent quark mass $m=400 \mathrm{MeV}$.

the large scale. The fact that in that calculation the evolution effects are more pronounced than in the present approach is caused by the significantly lower scale $\left(\mu_{\text {bag }}=0.08 \mathrm{GeV}^{2}\right)$ used in ref [5]. On the other hand our results are quite different to those obtained in the QCD sum rule approach of ref [43]. The sum rule approach essentially predicts $h_{T}$ to be constant in the interval $0.3<x<0.8$. For small values of $x$ the authors of ref [43] assume a Regge behavior. In the (covariant) constituent quark model of Suzuki and Shijetamin 38 a result similar to ours is obtained when effects attributed to Goldstone bosons are included. Otherwise the maximum of their distribution is about $50 \%$ larger than in our calculation. These authors also observe that in magnitude the down quark component is significantly smaller than the up quark piece. The chiral chromo-dielectric model of Barone et al. [6] predicts a similar shape for $h_{T}$ but their distribution $h_{T}^{(u)}$ is larger than the one in NJL chiral soliton model. This is also reflected by the sizable value for the isovector tensor charge $\Gamma_{T}^{V}\left(Q^{2}=25 \mathrm{GeV}^{2}\right)=1.22$ and $\Gamma_{T}^{V}\left(Q^{2}=0.16 \mathrm{GeV}^{2}\right)=1.53$ in that approach. In the Isgur Karl model (which has $h_{1}=g_{1}$ ) the maximal value of $h_{1}$ is only about have as big as in our calculation, $c f$. figure 1 in ref [ $[$ ].

For completeness we also demonstrate in figs $\mathbf{9}$ and 10 that at the low model scale $Q_{0}^{2}=0.4 \mathrm{GeV}^{2}$ Soffer's inequality [4] is satisfied. This inequality relates the nucleon chiral 
$\mathbf{h}_{\mathrm{T}}^{\mathrm{n}}\left(\mathbf{x}, \mathbf{Q}^{2}\right)$

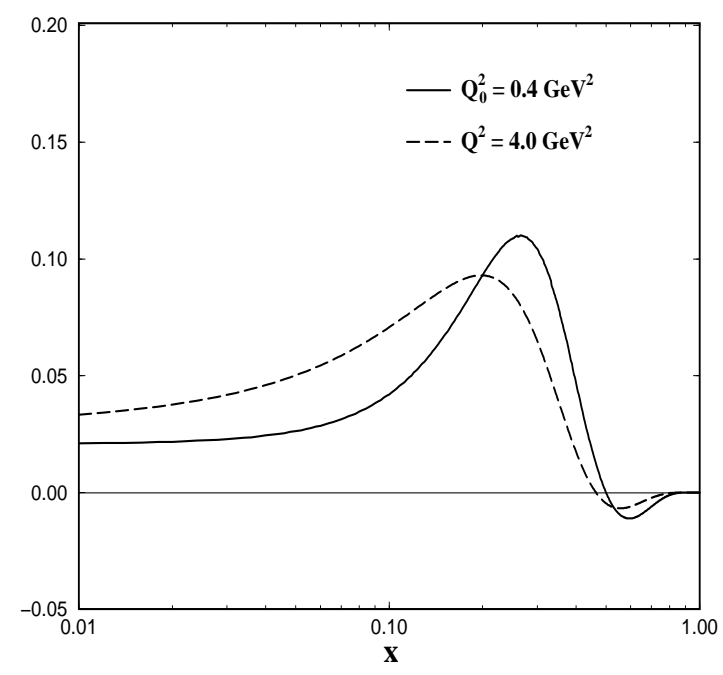

$\mathbf{h}_{\mathrm{L}}^{\mathrm{n}(2)}\left(\mathbf{x}, \mathbf{Q}^{2}\right)$

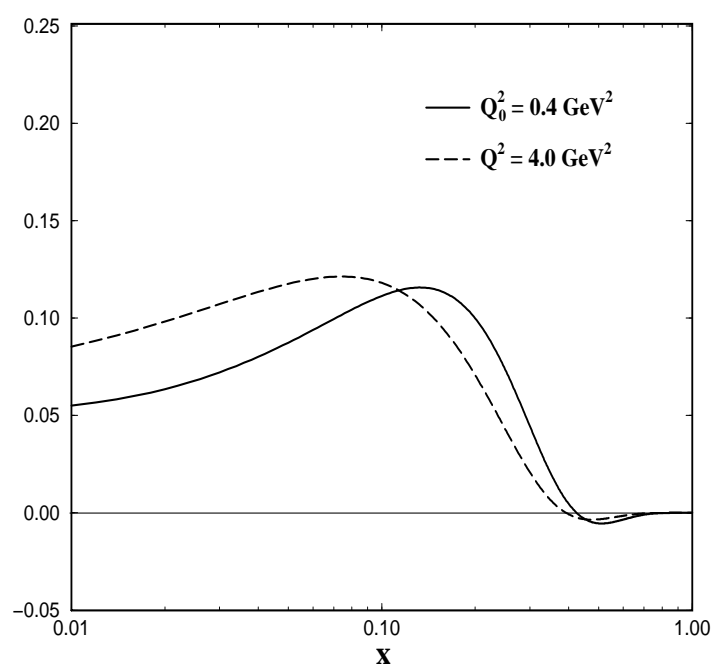

Figure 7: Left panel:The evolution of $h_{T}^{n}\left(x, Q^{2}\right)$ from $Q_{0}^{2}=0.4 \mathrm{GeV}^{2}$ (solid line) to $Q^{2}=4 \mathrm{GeV}^{2}$ (long-dashed line) for the constituent quark mass $m=400 \mathrm{MeV}$. Right panel: The evolution of the twist-2 contribution to the longitudinal chiral odd structure function, $h_{L}^{n(2)}\left(x, Q^{2}\right)$ from $Q_{0}^{2}=0.4 \mathrm{GeV}^{2}$ (solid line) to $Q^{2}=4 \mathrm{GeV}^{2}$ (long-dashed line) for $m=400 \mathrm{MeV}$.

odd distribution functions to both the unpolarized $f_{1}^{(q)}\left(x, Q_{0}^{2}\right)$ and polarized $g_{1}^{(q)}\left(x, Q_{0}^{2}\right)$ structure functions

$$
f_{1}^{(q)}\left(x, Q^{2}\right)+g_{1}^{(q)}\left(x, Q^{2}\right) \geq 2\left|h_{T}^{(q)}\left(x, Q^{2}\right)\right|
$$

Here the superscript refers to the flavor combination which projects onto up and down quark quantum numbers $(q=u, d)$. Note again, that this projection refers to the constituent quarks which contain some non-perturbative gluonic distributions. In figure 10 we display the down quark component of the inequality (34). As this component of $h_{T}$ is almost negligible the inequality is satisfied by the unpolarized structure function $f_{1}^{(d)}$ being larger in magnitude than the polarized one $g_{1}^{(d)}$. For the constituent quark mass $m=450 \mathrm{MeV}$ we also find that Soffer's inequality is satisfied at the model scale $Q_{0}$. The only remarkable difference to $m=400 \mathrm{MeV}$ is that the down quark component of $h_{T}^{(d)}$ does not possess nodes. This can already be inferred from figures 1 and 2 .

A more thorough model study of Soffer's inequality for scales other than $Q_{0}^{2}$ which also contains the next-to-leading order contributions in the evolution program is subject to further investigations 45]. The next-to-leading order calculation for $h_{T}$ \& in the Isgur-Karl model indicates that its scale dependence is slightly mitigated by the inclusion of next-to-leading order contributions. 

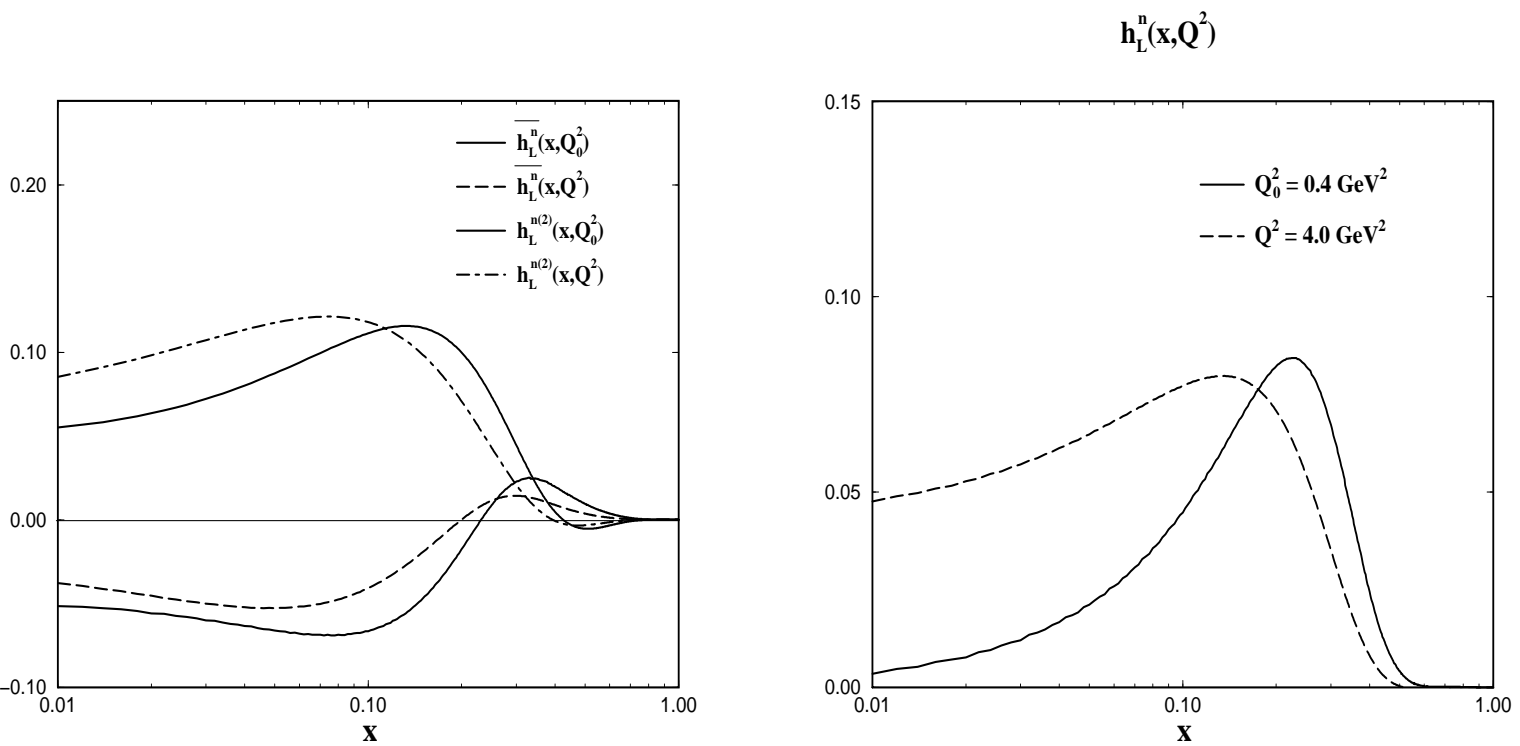

Figure 8: Left panel: The evolution of the twist-3 contribution to the longitudinal chiral odd structure function, $\bar{h}_{L}^{n}\left(x, Q^{2}\right)$ along with the corresponding twist-2 piece, $h_{L}^{n(2)}\left(x, Q^{2}\right)$. Right panel: The evolution of $h_{L}^{n}\left(x, Q^{2}\right)=h_{L}^{n(2)}\left(x, Q^{2}\right)+\bar{h}_{L}^{n}\left(x, Q^{2}\right)$ from $Q_{0}^{2}=0.4 \mathrm{GeV}^{2}$ (solid line) to $Q^{2}=4 \mathrm{GeV}^{2}$ (long-dashed line) for the constituent quark mass $m=400 \mathrm{MeV}$.

\section{Conclusions}

In this paper we have presented the NJL chiral soliton model calculation of the leading twist parts of the transverse and longitudinal chiral odd structure (distribution) functions of the nucleon. Data on these distribution functions should eventually be available from DIS experiments in the fragmentation regions (in conjunction with fragmentation functions) or be extracted from Drell-Yan experiments. These structure functions serve to complete our picture of the spin distributions of the nucleon. The most important feature of the present quark based model is that it is chirally invariant and that this symmetry is dynamically broken. After bosonization the NJL model becomes an effective meson theory in which baryons emerge as self-consistent soliton solutions exactly the way as expected from large $N_{C}$ considerations in QCD. Chiral soliton models are particularly interesting in the context of the nucleon spin structure as these models nicely explain the small contribution of the quarks the total nucleon spin.

In the NJL chiral soliton model there are two contributions to nucleon properties. First, there is the contribution of the distinct valence quark level. This is the lowest level in the quark spectrum and bound in the background of the chiral soliton. Second, there 

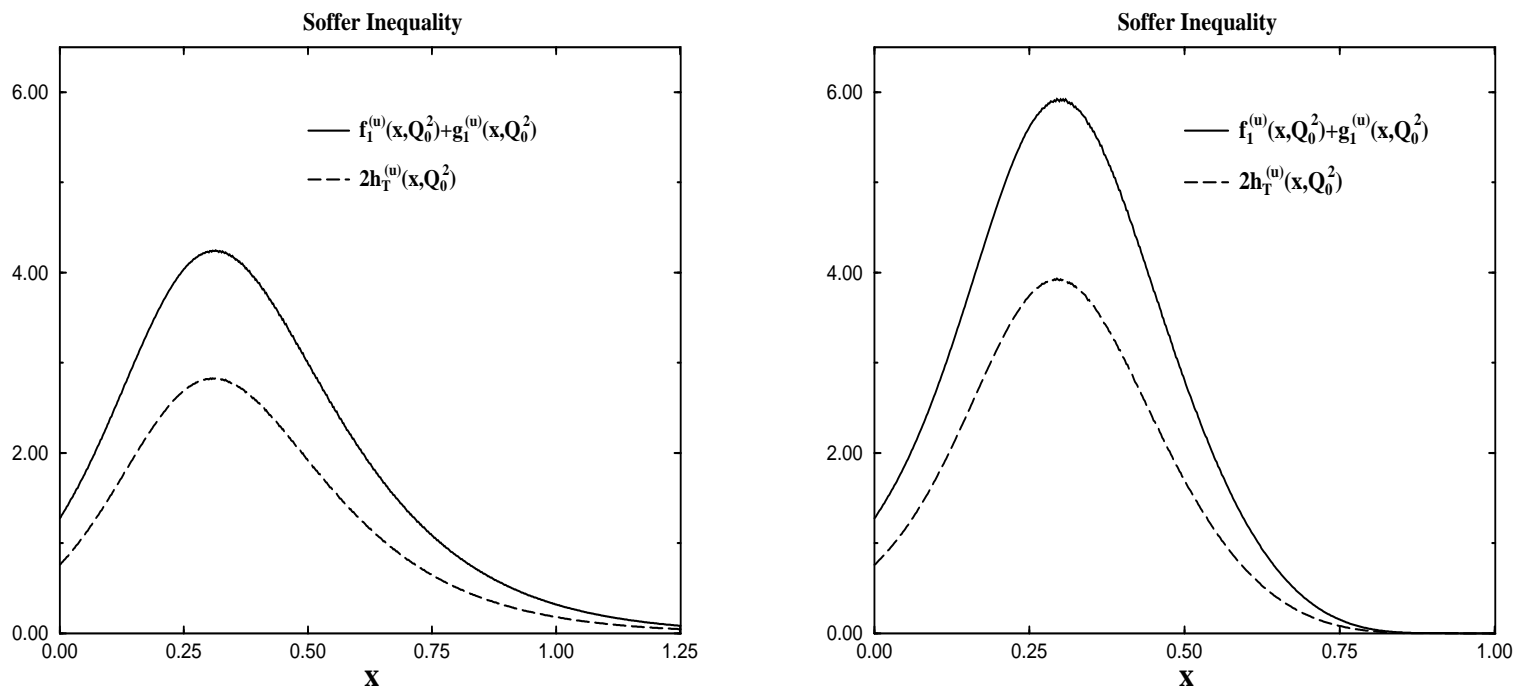

Figure 9: Left panel (9a): The Soffer inequality for the chiral even combination $f_{1}^{(u)}\left(x, Q_{0}^{2}\right)+g_{1}^{(u)}\left(x, Q_{0}^{2}\right)$ (solid line) of the effective up-quark distributions and the chiral odd structure function $2 h_{T}^{(u)}\left(x, Q_{0}^{2}\right)$ (long-dashed line) for a constituent quark mass of $m=400 \mathrm{MeV}$, calculated in the nucleon rest frame (RF). Right panel: (9b) Same as figure ga calculated in the infinite momentum frame (IMF). The transformation prescription is given in eq (26).

is the part which is associated to the polarization (by the soliton) of the vacuum. For many static nucleon properties the latter contribution is quite small, in particular for those which are related to the axial (spin) properties of the nucleon. This is a strong indication that the vacuum contribution to the chiral odd structure functions is negligible as well. Hence it seems more important to include substantial $1 / N_{C}$ corrections to the valence quark contribution. These corrections come about when projecting the soliton onto states with good spin and isospin, i.e. proton and neutron. Inclusion of these $1 / N_{C}$ corrections together with a consistently regularized treatment of the vacuum polarization is technically rather involved and beyond the scope of the present paper. The numerical results for the tensor charge indicate that the vacuum contributions are in fact negligibly small.

When the model structure functions are computed one immediately recognizes that they have improper support due to the breaking of translational invariance by the background soliton, i.e. the structure functions do not exactly vanish for $x>1$. This can be cured by Lorentz boosting to the infinite momentum frame which is particularly suited for DIS processes. Although the un-boosted structure functions are negligibly small at 

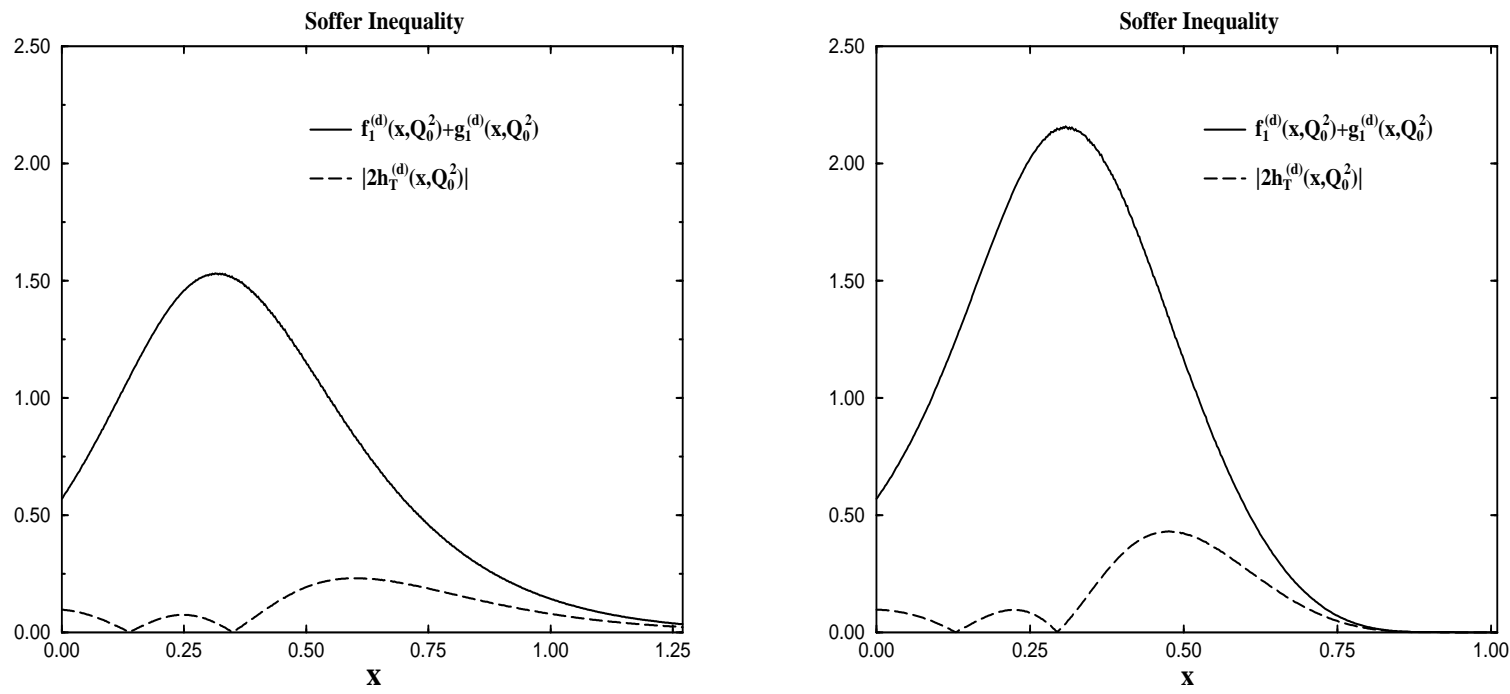

Figure 10: Same as figure 9 for the down quark combination.

$x>1$ the transformation to this frame is essential and has sizable effects on the structure functions at moderate $x$. However, the most important issue when comparing the model predictions to (not yet available) experimental data is the observation that the model represents QCD at a low momentum scale $Q_{0}^{2}$. A priori this scale represents an additional parameter to the model calculation which, for consistency, has to be smaller than the ultraviolet cut-off of the model $\Lambda^{2}=0.56 \mathrm{GeV}^{2}$. For the model under consideration we previously fixed $Q_{0}^{2}$ when studying the unpolarized structure functions and found $Q_{0}^{2}=0.4 \mathrm{GeV}^{2}$. The important logarithmic corrections to the model structure functions are then obtained within a generalized GLAP evolution program. In this context we have restricted ourselves to a leading order (in $\alpha_{\mathrm{QCD}}$ ) calculation because the anomalous dimensions, which govern the QCD evolution, for the twist-3 piece of the longitudinal part of the chiral odd structure are only known to that order. As the full evolution to the longitudinal structure function involves both twist-2 and twist- 3 pieces this restriction is consistent. We have seen that the QCD evolution of the chiral odd structure function leads to sizable enhancements at low $x$, i.e. in the region $0.01 \leq x \leq 0.10$. In this respect the present situation is similar to that for the polarized structure functions. A difference to the polarized structure function is that the lowest moment is not protected against logarithmic corrections, even at leading order in $\alpha_{\mathrm{QCD}}$. For the nucleon tensor charge we thus find a reduction of about $10 \%$ upon evolution to $Q^{2}=4.0 \mathrm{GeV}^{2}$. We have also compared the neutron and proton chiral odd structure functions. This has been achieved 
by the inclusion of the $1 / N_{C}$ cranking corrections. In absolute value the proton structure functions are about twice as large as those of the neutron. Furthermore the neutron structure functions drop to zero at a lower value of $x$. These two effects can be linked to the down quark component of the transverse nucleon chiral odd distribution functions being significantly smaller than the component with up-quark quantum numbers. We have also observed that neither of these features is effected by the evolution program.

\section{Acknowledgements}

This work has been supported in part by the Deutsche Forschungsgemeinschaft (DFG) under contract Re 856/2-3, and by the U.S. Department of Energy (D.O.E.) under contract DE-FE-02-95ER40923. One of us (LG) is grateful to G. R. Goldstein for helpful comments and to K. A. Milton for encouragement and support.

\section{A Bilocal Light Cone Distributions}

In this appendix we outline the steps giving rise to the starting point of our calculation, eqs (14) and (15) in section 3.

It is well known that the chiral odd spin-dependent structure functions $h_{T}(x)$ and $h_{L}(x)$ do not contribute to the hadronic tensor in DIS. $h_{T}(x)$ was first studied by Ralston and Soper [1] in the context of polarized Drell-Yan processes while $h_{L}(x)$ was more recently detailed by Jaffe and $\mathrm{Ji}$ [4]. In the latter study a general Lorentz decomposition of invariant matrix elements of the characteristic bilocal operators in hard processes, $\bar{\Psi}(0) \Gamma_{\mu} \Psi(\lambda n)$ was performed. Adopting light-cone variables reveals that up to twist-3 there are six invariant structure functions that characterize the nucleon. The leading twist (two and three) spin-dependent contributions emerging from the Lorentz decomposition of these hard processes are given by [3],

$$
\begin{aligned}
\int \frac{d \lambda}{2 \pi} e^{i \lambda x}\left\langle P, S\left|\bar{\Psi}(0) i \sigma_{\mu \nu} \gamma_{5} \Psi(\lambda n)\right| P, S\right\rangle= & 2\left\{h_{T}(x)\left(S_{\perp \mu} p_{\nu}-S_{\perp \nu} p_{\mu}\right) / M\right. \\
& \left.+h_{L}(x) M\left(p_{\mu} p_{\nu}-p_{\nu} p_{\mu}\right) S \cdot n\right\}
\end{aligned}
$$

\footnotetext{
${ }^{7}$ See ref [43] for the definition of chiral odd structure functions in the language of a hadronic tensor. Actually such a definition is sufficient to carry over the QCD definition of the chiral odd structure functions to the NJL model because formally the NJL model currents are identically to those in QCD.
} 


$$
\int \frac{d \lambda}{2 \pi} e^{i \lambda x}\left\langle p, S\left|\Psi(0) i \gamma_{\mu} \gamma_{5} \Psi(\lambda n)\right| p, S\right\rangle=2\left\{g_{L}(x) p_{\mu} S \cdot n+g_{T}(x) S_{\perp \mu}\right\}
$$

where $p^{\mu}$ and $n^{\mu}$ define a light-like coordinate system, i.e. $p \cdot n=1, p^{2}=n^{2}=0$ and $|P S\rangle$ denotes the nucleon state with four momentum $P$ and spin $S$. In the system where the nucleon is moving along the $\hat{\boldsymbol{z}}$ direction one conveniently defines the four vectors

$$
\begin{aligned}
p^{\mu} & =\frac{\mathcal{P}}{\sqrt{2}}(1,0,0,1) \\
n^{\mu} & =\frac{1}{\sqrt{2}} \mathcal{P}(1,0,0,-1) .
\end{aligned}
$$

In this system the nucleon momentum is given by $P^{\mu}=p^{\mu}+M n^{\mu} / 2$ and spin $S^{\mu}$ is decomposed as $S^{\mu}=(S \cdot n) p^{\mu}+(S \cdot p) n^{\mu}+S_{\perp}^{\mu}$. Finally, $\mathcal{P} \rightarrow \infty$ corresponds to the infinite momentum frame (IMF) and $\mathcal{P}=M / \sqrt{2}$ corresponds to the nucleon rest frame $(\mathrm{RF})$. Utilizing the convenient projection properties of the light-like vectors the defining equation (35) may be inverted. One obtains for the chiral odd structure functions

$$
h_{T}(x)=\frac{1}{M} \int \frac{d \lambda}{2 \pi} e^{i \lambda x}\left\langle P S_{\perp}\left|\Psi_{+}^{\dagger}(0) \gamma_{\perp} \gamma_{5} \Psi_{+}(\lambda n)\right| P, S_{\perp}\right\rangle
$$

and

$$
\begin{array}{r}
h_{L}(x)=\frac{1}{2 M} \int \frac{d \lambda}{2 \pi} e^{i \lambda x}\left\langle P S_{z}\right| \Psi_{-}^{\dagger}(0) \gamma_{0} \gamma_{5} \Psi_{+}(\lambda n) \\
-\Psi_{+}^{\dagger}(0) \gamma_{0} \gamma_{5} \Psi_{-}(\lambda n)\left|P, S_{z}\right\rangle .
\end{array}
$$

The quark bilocals describe the propagation of the intermediate constituent quark which is struck by the external source. The forward propagation is described by $x \geq 0$ while negative $x$ parameterize an intermediate quark which moves backward. In what follows we will only consider positive $x$ in conjunction with the contribution associated with the forward propagating quark $h^{(+)}(x)$. The backward contribution can easily be obtained from $h^{(+)}(-x)$. Finally, noting the change of variables from light-like coordinates $\left(\eta, \lambda, \boldsymbol{\xi}_{\perp}\right)$ to light-cone coordinates $\left(\xi^{+}, \xi^{-}, \boldsymbol{\xi}_{\perp}\right)$ where in particular,

$$
\xi^{+}=\eta \mathcal{P} \quad \text { and } \quad \xi^{-}=\frac{\lambda}{\mathcal{P}}
$$

yields the chiral odd quark transverse distributions,

$$
\begin{aligned}
h_{T}^{(+)}(x)=\frac{\sqrt{2}}{4 \pi} \int & d \xi^{-} \exp \left(-i \xi^{-} \frac{M x}{\sqrt{2}}\right) \\
& \times\left\langle\boldsymbol{S}_{\perp}\left|\Psi_{+}^{\dagger}(\xi) \gamma_{\perp} \gamma_{5} \Psi_{+}(0)\right| \boldsymbol{S}_{\perp}\right\rangle_{\xi^{+}=\boldsymbol{\xi}_{\perp}=0}
\end{aligned}
$$


and the longitudinal contribution,

$$
\begin{aligned}
h_{L}^{(+)}(x)=\frac{\sqrt{2}}{8 \pi} & \int d \xi^{-} \exp \left(-i \xi^{-} \frac{M x}{\sqrt{2}}\right) \\
& \times\left\langle\boldsymbol{S}_{z}\left|\Psi_{+}^{\dagger}(\xi) \gamma_{0} \gamma_{5} \Psi_{-}(0)-\Psi_{-}^{\dagger}(\xi) \gamma_{0} \gamma_{5} \mathcal{Q}^{2} \Psi_{+}(0)\right| \boldsymbol{S}_{z}\right\rangle_{\xi^{+}=\boldsymbol{\xi}_{\perp}=0} .
\end{aligned}
$$

These equations represent the starting point of section 3 .

\section{B Chiral Odd Structure Functions in the NJL Soli- ton Model}

In this appendix we derive and summarize the explicit expressions for the chiral odd structure functions, eqs (24). The first step is to construct the eigenfunctions of the single particle Dirac Hamiltonian (8) in coordinate space. The hedgehog ansatz (7) connects coordinate space with isospace and these eigenfunctions are also eigenstates of the grand spin operator

$$
\boldsymbol{G}=\boldsymbol{J}+\frac{\boldsymbol{\tau}}{2}=\boldsymbol{l}+\frac{\boldsymbol{\sigma}}{2}+\frac{\boldsymbol{\tau}}{2}
$$

which is the sum of the total spin $\boldsymbol{J}$ and the isospin $\boldsymbol{\tau} / 2$. The spin itself is decomposed into orbital angular momentum $\boldsymbol{l}$ and intrinsic spin $\boldsymbol{\sigma} / 2$. Denoting by $M$ the grand spin projection quantum number the tensor spherical harmonics which are associated with the grand spin may be written as $\mathcal{Y}_{l, j}^{G, M}(\hat{\boldsymbol{r}})$. Note that these tensor spherical harmonics are two-component spinors in both spin and isospin spaces. Given a profile function $\Theta(r)$ the numerical diagonalization of the Dirac Hamiltonian (8) yields the radial functions $g_{\mu}^{(G,+, 1)}(r), f_{\mu}^{(G,+, 1)}(r)$, etc. in the decomposition (cf. ref 46])

$$
\begin{aligned}
& \Psi_{\mu}^{(G,+)}(\boldsymbol{r})=\left(\begin{array}{c}
i g_{\mu}^{(G,+; 1)}(r) \mathcal{Y}_{G, G+\frac{1}{2}}^{G, M}(\hat{\boldsymbol{r}}) \\
f_{\mu}^{(G,+; 1)}(r) \mathcal{Y}_{G, M}^{G, G+\frac{1}{2}}(\hat{\boldsymbol{r}})
\end{array}\right)+\left(\begin{array}{c}
i g_{\mu}^{(G,+; 2)}(r) \mathcal{Y}_{G, G-\frac{1}{2}}^{G, M}(\hat{\boldsymbol{r}}) \\
-f_{\mu}^{(G,+; 2)}(r) \mathcal{Y}_{G-1, G-\frac{1}{2}}^{G, M}(\hat{\boldsymbol{r}})
\end{array}\right) \\
& \Psi_{\mu}^{(G,-)}(\boldsymbol{r})=\left(\begin{array}{c}
i g_{\mu}^{(G,-; 1)}(r) \mathcal{Y}_{G, M}^{G, M}(\hat{\boldsymbol{r}}) \\
-f_{\mu}^{(G,-; 1)}(r) \mathcal{Y}_{G, G+\frac{1}{2}}^{G, M}(\hat{\boldsymbol{r}})
\end{array}\right)+\left(\begin{array}{c}
i g_{\mu}^{(G,-; 2)}(r) \mathcal{Y}_{G, M}^{G, M}(\hat{\boldsymbol{r}}) \\
f_{\mu}^{(G,-; 2)}(r) \mathcal{Y}_{G, G-\frac{1}{2}}^{G, M}(\hat{\boldsymbol{r}})
\end{array}\right) .
\end{aligned}
$$

The second superscript $( \pm)$ denotes the intrinsic parity, which also is a conserved quantum number. 8 Note that for the $G=0$ channel, which contains the mean-field contribution to the valence quark wave-function in eq (13)

$$
\Psi_{\mathrm{v}}(\boldsymbol{r})=\left(\begin{array}{c}
i g_{\mathrm{v}}(r) \mathcal{Y}_{0, \frac{1}{2}}^{0,0}(\hat{\boldsymbol{r}}) \\
f_{\mathrm{v}}(r) \mathcal{Y}_{1, \frac{1}{2}}^{0,0}(\hat{\boldsymbol{r}})
\end{array}\right),
$$

\footnotetext{
${ }^{8}$ The total parity is given by the product of the intrinsic parity and $(-)^{G}$.
} 
only the components with $j=+1 / 2$ are allowed. In addition to this mean-field piece (46) the complete valence quark wave-function (13) also contains the cranking correction, which dwells in the channel with $G=1$ and negative intrinsic parity.

The discretization $(\mu)$ is accomplished by choosing suitable boundary conditions at a radial distance which is large compared to the soliton extension [46, 47]. This calculation yields the energy eigenvalues $\epsilon_{\mu}$, which enter the energy functional (9). The soliton configuration is finally determined by self-consistently minimizing this energy functional. In ref [48] the numerical procedure is described in detail.

We continue by making explicit the Fourier transform of eq (13),

$$
\tilde{\psi}_{\mathrm{v}}(\boldsymbol{p})=\int \frac{d^{3} x}{4 \pi} \psi_{\mathrm{v}}(\boldsymbol{x}) \exp (i \boldsymbol{p} \cdot \boldsymbol{x})=\tilde{\Psi}_{\mathrm{v}}(\boldsymbol{p})+Q_{\mu} \tilde{\Psi}_{\mu}(\boldsymbol{p})
$$

The leading order in $N_{C}$ valence quark contribution is just the Fourier transform of (46)

$$
\tilde{\Psi}_{\mathrm{v}}(\boldsymbol{p})=i\left(\begin{array}{cc}
\tilde{g}_{\mathrm{v}}(p) & \mathcal{Y}_{0, \frac{1}{2}}^{0,0}(\hat{\boldsymbol{p}}) \\
\tilde{f}_{\mathrm{v}}(p) & \mathcal{Y}_{1, \frac{1}{2}}^{0,0}(\hat{\boldsymbol{p}})
\end{array}\right)
$$

and the cranking correction involves the Fourier transform of spinor with $G=1$ and negative intrinsic parity

$$
\tilde{\Psi}_{\mu}(\boldsymbol{p})=-i\left(\begin{array}{c}
\tilde{g}_{\mu}^{(1)}(p) \mathcal{Y}_{2, \frac{3}{2}}^{1, M}(\hat{\boldsymbol{p}})-\tilde{g}_{\mu}^{(2)}(p) \mathcal{Y}_{0, \frac{1}{2}}^{1, M}(\hat{\boldsymbol{p}}) \\
\tilde{f}_{\mu}^{(1)}(p) \mathcal{Y}_{1, \frac{3}{2}}^{1, M}(\hat{\boldsymbol{p}})-\tilde{f}_{\mu}^{(2)}(p) \mathcal{Y}_{1, \frac{1}{2}}^{1, M}(\hat{\boldsymbol{p}})
\end{array}\right)
$$

Here $\mathcal{Y}_{l, j}^{G, M}(\hat{\boldsymbol{p}})$ are the Fourier transforms of the tensor spherical harmonics associated with the grand spin operator (43). The Fourier transform for the radial functions in eqs (48) and (49) is defined by

$$
\tilde{\phi}_{\mu}(p)=\int_{0}^{R} d r r^{2} j_{l}(p r) \phi_{\mu}(r)
$$

Here the index $l$ of the spherical Bessel function denotes the orbital angular momentum of the associated tensor spherical harmonic. We have suppressed the grand spin index on the transforms of the radial wave functions for convenience. For purposes of notation we have also introduced the quantity $Q_{\mu}$ in eq (47) which parameterizes the cranking corrections in eq (13)

$$
\begin{aligned}
\frac{\langle\mu|\tau \cdot \Omega| \mathrm{v}\rangle}{\epsilon_{\mathrm{v}}-\epsilon_{\mu}} & =\alpha^{2} Q_{\mu}\left\{\frac{\delta_{M, 1}}{\sqrt{2}}\left(\Omega_{1}+i \Omega_{2}\right)-\frac{\delta_{M,-1}}{\sqrt{2}}\left(\Omega_{1}-i \Omega_{2}\right)-\delta_{M, 0} \Omega_{0}\right\} \delta_{G_{\mu}, 1} \\
& =Q_{\mu}\left\{\frac{\delta_{M, 1}}{\sqrt{2}}\left(J_{1}+i J_{2}\right)-\frac{\delta_{M,-1}}{\sqrt{2}}\left(J_{1}-i J_{2}\right)-\delta_{M, 0} J_{0}\right\} \delta_{G_{\mu}, 1}
\end{aligned}
$$


where

$$
Q_{\mu} \equiv \frac{1}{\alpha^{2}\left(\epsilon_{\mathrm{v}}-\epsilon_{\mu}\right)} \int d r r^{2}\left\{g_{\mathrm{v}}(r) g_{\mu}^{(2)}(r)+f_{\mathrm{v}}(r) f_{\mu}^{(2)}(r)\right\}
$$

In this definition we have included the total moment of inertia $\alpha^{2}$. In the proper-time regularization of the NJL chiral soliton model $\alpha^{2}$ is given by 19

$$
\begin{aligned}
\alpha_{\mathrm{v}}^{2} & =\frac{N_{C}}{2} \sum_{\mu \neq \mathrm{v}} \frac{\left|\left\langle\mathrm{v}\left|\tau_{3}\right| \mu\right\rangle\right|^{2}}{\left(\epsilon_{\mu}-\epsilon_{\mathrm{v}}\right)}, \\
\alpha_{\mathrm{s}}^{2} & =\frac{N_{C}}{4 \alpha^{2}} \sum_{\mu \nu} f_{\mu \nu}(\Lambda)\left\langle\mu\left|\tau^{3}\right| \nu\right\rangle\left\langle\nu\left|\tau^{3}\right| \mu\right\rangle, \\
\alpha^{2} & =\frac{1}{2}\left(1+\operatorname{sgn}\left(\epsilon_{\mathrm{val}}\right)\right) \alpha_{\mathrm{v}}^{2}+\alpha_{\mathrm{s}}^{2} .
\end{aligned}
$$

The regulator function in the vacuum contribution reads

$$
f_{\mu \nu}(\Lambda)=\frac{\Lambda}{\sqrt{\pi}} \frac{e^{-\left(\epsilon_{\mu} / \Lambda\right)^{2}}-e^{-\left(\epsilon_{\nu} / \Lambda\right)^{2}}}{\epsilon_{\nu}^{2}-\epsilon_{\mu}^{2}}-\frac{\operatorname{sgn}\left(\epsilon_{\nu}\right) \operatorname{erfc}\left(\left|\frac{\epsilon_{\nu}}{\Lambda}\right|\right)-\operatorname{sgn}\left(\epsilon_{\mu}\right) \operatorname{erfc}\left(\left|\frac{\epsilon_{\mu}}{\Lambda}\right|\right)}{2\left(\epsilon_{\mu}-\epsilon_{\nu}\right)} .
$$

The moment of inertia enters via the quantization description for the collective coordinates $\Omega \rightarrow \alpha^{2} \boldsymbol{J}$ with $\boldsymbol{J}$ being the nucleon spin operator. In this quantization prescription we had previously restricted the moment of inertia to its valence quark contribution, $\alpha_{\mathrm{v}}^{2}$, to ensure that the Adler sum rule for the unpolarized structure functions is maintained in the valence quark approximation [17]. For small or moderate constituent quark masses the valence contribution to the moment of inertia is about $80 \%$ or more [47]. This is one of the reasons to believe that the valence quark approximation to structure functions is sensible. In the case of the chiral odd structure functions (as for the polarized ones) the valence quark approximation appears to be even better. As we see from table 1 the lowest moments of these structure functions are saturated to about $95 \%$ by the valence quark contribution. Hence it is reasonable to assume that the vacuum contribution to these structure functions in negligibly small. As a consequence the valence quark approximation with the total moment of inertia substituted into the quantization rule will provide a very reliable estimate of the chiral odd structure functions.

Together with $\left\langle N\left|D_{i j}\right| N\right\rangle=-(4 / 3) I_{i} J_{j}$ 13 the nucleon matrix elements may now easily be computed. Here $\boldsymbol{I}$ denotes the nucleon isospin. Whenever products of collective coordinates and operators appear which do not commute after canonical quantization we adopt the symmetric ordering. This is consistent with fundamental requirements such as PCAC. Defining finally the following combinations

$$
\tilde{f}^{(i)}(p)=Q_{\mu} \tilde{f}_{\mu}^{(i)}(p) \quad \text { and } \quad \tilde{g}^{(i)}(p)=Q_{\mu} \tilde{g}_{\mu}^{(i)}(p),
$$


for $i=1,2$. The isoscalar(vector) contributions to the chiral odd structure functions (24) read

$$
\begin{aligned}
& h_{T, \pm}^{I=0}\left(x, \mu^{2}\right)=N_{C} \frac{5 M_{N}}{36 \pi} \int_{M_{N}\left|x_{\mp}\right|}^{\infty} p d p \\
& \times\left\{\tilde{g}_{\mathrm{v}}(p) \tilde{g}^{(1)}(p) \frac{3 \cos ^{2}\left(\theta_{p}^{ \pm}\right)-1}{4 \sqrt{2}}-\frac{1}{2} \tilde{g}_{\mathrm{v}}(p) \tilde{g}^{(2)}(p)\right. \\
& \pm\left(\tilde{g}_{\mathrm{v}}(p) \tilde{f}^{(1)}(p)+\tilde{f}_{\mathrm{v}}(p) \tilde{g}^{(1)}(p)\right) \frac{\cos \left(\theta_{p}^{ \pm}\right)}{\sqrt{8}} \\
& \mp\left(\tilde{f}_{\mathrm{v}}(p) \tilde{g}^{(2)}(p)+\tilde{g}_{\mathrm{v}}(p) \tilde{f}^{(2)}(p)\right) \frac{\cos \left(\theta_{p}^{ \pm}\right)}{2} \\
& \left.-\tilde{f}_{\mathrm{v}}(p) \tilde{f}^{(1)}(p) \frac{\cos ^{2}\left(\theta_{p}^{ \pm}\right)-3}{4 \sqrt{2}}-\tilde{f}_{\mathrm{v}}(p) \tilde{f}^{(2)}(p) \frac{\cos \left(\theta_{p}^{ \pm}\right) 2}{2}\right\} \\
& h_{T, \pm}^{I=1}\left(x, \mu^{2}\right)=N_{C} \frac{M_{N}}{36 \pi} \int_{M_{N}\left|x_{\mp}\right|}^{\infty} p d p \\
& \times\left\{\tilde{g}_{\mathrm{v}}(p)^{2} \pm 2 \tilde{g}_{\mathrm{v}}(p) \tilde{f}_{\mathrm{v}}(p) \cos \left(\theta_{p}^{ \pm}\right)+\tilde{f}_{\mathrm{v}}(p)^{2}\left(\cos ^{2}\left(\theta_{p}^{ \pm}\right)\right)\right\} \\
& h_{L, \pm}^{I=0}\left(x, \mu^{2}\right)=N_{C} \frac{5 M_{N}}{36 \pi} \int_{M_{N}\left|x_{\mp}\right|}^{\infty} p d p \\
& \times\left\{ \pm \tilde{g}_{\mathrm{v}}(p) \tilde{g}^{(1)}(p) \frac{3 \cos ^{2}\left(\theta_{p}^{ \pm}\right)-1}{4 \sqrt{2}} \pm \frac{1}{2} \tilde{g}_{\mathrm{v}}(p) \tilde{g}^{(2)}(p)\right. \\
& \left.\mp \tilde{f}_{\mathrm{v}}(p) \tilde{f}^{(1)}(p) \frac{1+\cos ^{2}\left(\theta_{p}^{ \pm}\right)}{2 \sqrt{2}} \mp \tilde{f}_{\mathrm{v}}(p) \tilde{f}^{(2)}(p) \frac{2 \cos ^{2}\left(\theta_{p}^{ \pm}\right)-1}{2}\right\} \text {, } \\
& h_{L, \pm}^{I=1}\left(x, \mu^{2}\right)=-N_{C} \frac{M_{N}}{36 \pi} \int_{M_{N}\left|x_{\mp}\right|}^{\infty} p d p\left\{\mp \tilde{g}_{\mathrm{v}}(p)^{2} \pm \tilde{f}_{\mathrm{v}}(p)^{2}\left(2 \cos ^{2}\left(\theta_{p}^{ \pm}\right)-1\right)\right\}
\end{aligned}
$$

which we evaluate numerically. Note that the angle $\theta_{p}^{ \pm}$is related to the integration variable $p$ via

$$
\cos \theta_{p}^{ \pm}=\frac{1}{p}\left|M_{N} x \pm \epsilon_{\mathrm{v}}\right|
$$

In ref [10] the contribution to structure function $h_{T}$ from effective quark distributions was calculated omitting the cranking corrections and adopting an external (non-selfconsistent) meson profile.

\footnotetext{
${ }^{9}$ In this work it is important to note that the quark distributions refer to constituent quarks, $m_{q} \approx$ $400 \mathrm{MeV}$, it is thus misleading to compare them with the data of parton distributions from either DrellYan or DIS processes.
} 


\section{Evolution of $\bar{h}_{L}\left(x, Q^{2}\right)$}

In this appendix we outline our technique to evolve the low scale model prediction for the twist-3 piece $\bar{h}_{L}\left(x, Q_{0}^{2}\right)$ to the larger scale $Q^{2}$. This utilizes the method described in refs [9, 49] based on the results of ref [50]. The $Q^{2}$ evolution of the moments

$$
\mathcal{M}_{n}\left[\bar{h}_{L}\left(Q^{2}\right)\right]=\int_{0}^{1} d x x^{n} \bar{h}_{L}\left(x, Q^{2}\right)
$$

is given by

$$
\mathcal{M}_{n}\left[\bar{h}_{L}\left(Q^{2}\right)\right]=\left(\frac{\alpha\left(Q^{2}\right)}{\alpha\left(Q_{0}^{2}\right)}\right)^{\gamma_{n}^{h} / b_{0}} \mathcal{M}_{n}\left[\bar{h}_{L}\left(Q_{0}^{2}\right)\right]
$$

Here $b_{0}=\left(11 N_{C}-2 n_{f}\right) / 3$ is coefficient of the leading term in the QCD-beta function. Also, $N_{C}$ and $n_{f}$ are the number of colors and flavors respectively. Within the $N_{C} \rightarrow \infty$ approximation the anomalous dimensions are [34]

$$
\gamma_{n}^{h}=2 N_{C}\left(S_{n}+\gamma_{\mathrm{E}}-\frac{1}{4}+\frac{3}{2(n+1)}\right)
$$

with $S_{n}=\sum_{j=1}^{n}(1 / j)-\gamma_{\mathrm{E}}$ where $\gamma_{E}=0.577 \ldots$ is the Euler constant which has been introduced for later convenience.

In order to find the QCD-evolution of the structure functions one needs to invert the Mellin-transform (64). This can be achieved by noting that the Bernstein polynomial

$$
b^{(N, k)}(x)=(N+1)\left(\begin{array}{l}
n \\
k
\end{array}\right) x^{k}(1-x)^{N-k}=\frac{(N+1) !}{k !} \sum_{l=0}^{N-k} \frac{(-1)^{l} x^{k+l}}{l !(N-k-l) !},
$$

has the property,

$$
\lim _{\substack{N, k \rightarrow \infty \\ k / N \rightarrow x}} b^{(N, k)}(y)=\delta(x-y)
$$

for $0<x, y<1$. This enables one to express the structure function via its moments

$$
\bar{h}_{L}\left(x, Q^{2}\right)=\lim _{\substack{N, k \rightarrow \infty \\ k / N \rightarrow x}} \frac{(N+1) !}{k !} \sum_{l=0}^{N-k} \frac{(-1)^{l}}{l !(N-k-l) !} \int_{0}^{1} d y y^{k+l} \bar{h}_{L}\left(y, Q^{2}\right)
$$

which depend on $Q^{2}$ as indicated in eq (65)

$$
\bar{h}_{L}\left(x, Q^{2}\right)=\lim _{\substack{N, k \rightarrow \infty \\ k / N \rightarrow x}} \frac{(N+1) !}{k !} \sum_{l=0}^{N-k} \frac{(-1)^{l}}{l !(N-k-l) !} L^{\gamma_{k+l}^{h} / b_{0}} \int_{0}^{1} d y y^{k+l} \bar{h}_{L}\left(y, Q_{0}^{2}\right) \text {. }
$$


Here $L=\alpha\left(Q^{2}\right) / \alpha\left(Q_{0}^{2}\right)$ denotes the ratio of the running coupling constants in QCD. Unfortunately, the rapid oscillations in the summation over $l$ in (70) due to the factor $(-1)^{l}$ preclude numerical summation of (70). Yet, observing that the expression $L^{\gamma_{n}^{h} / b_{0}}$ may be expanded as

$$
L^{\gamma_{n}^{h} / b_{0}}=a(L) \sum_{i=0} \frac{\mathcal{C}_{i}(L)}{(n+p)^{i-r(L)}},
$$

where $a(L)$, and $r(L)$ are constants determined from the asymptotic form $(n \rightarrow \infty)$ of eq (71)

$$
r(L)=2 N_{C} \ln (L) / b_{0} \quad \text { and } \quad a(L)=\exp \left[r(L)\left(\gamma_{\mathrm{E}}-\frac{1}{4}\right)\right]
$$

one can perform the sum to any desired accuracy. It should be noted that $p$ remains undetermined. It may be varied to control the convergence of the series (71). To determine the expansion coefficients $\mathcal{C}_{i}(L)$ we rearrange eq (71) to a Fourier expansion,

$$
(1-z p)^{r} \exp \left[\frac{1}{2 n}+\frac{3}{2 n+2}-\sum_{k=1}^{\infty}\left(\frac{\mathcal{B}_{2 k}}{2 k n^{2 k}}\right)\right]=\sum_{i=0}^{\infty} C_{i}(r) z^{i} .
$$

Here $z=1 /(p+n) \Leftrightarrow n=1 / z-p$ and $\mathcal{C}_{i}(L)=C_{i}(r)$. Furthermore we have utilized the asymptotic expansion of

$$
S_{n}=\ln (n)+\frac{1}{2 n}-\sum_{k=1}^{\infty}\left(\frac{\mathcal{B}_{2 k}}{2 k n^{2 k}}\right)
$$

where the $\mathcal{B}_{2 k}$ 's are the Bernoulli numbers. Performing a Taylor series to eighth order in $z$ yields the following values for the expansion coefficients $\mathcal{C}_{i}(L)=C_{i}(r(L)$ ) (for $p=2$ )

$$
\begin{aligned}
C_{0}(r) & =1, \quad C_{1}(r)=0, \quad C_{2}(r)=\frac{5}{12} r \\
C_{3}(r) & =\frac{1}{2} r, \quad C_{4}(r)=\frac{61}{120} r+\frac{25}{288} r^{2}, \quad C_{5}(r)=\frac{1}{2} r+\frac{5}{24} r^{2}, \\
C_{6}(r) & =\frac{125}{252} r+\frac{97}{288} r^{2}+\frac{125}{10368} r^{3}, \\
C_{7}(r) & =\frac{1}{2} r+\frac{37}{80} r^{2}+\frac{25}{576} r^{3}, \\
C_{8}(r) & =\frac{121}{240} r+\frac{354341}{604800} r^{2} \frac{665}{6912} r^{3}+\frac{625}{497664} r^{4}
\end{aligned}
$$

which gives more than adequate convergence of the series.

Finally we may write

$$
\bar{h}_{L}\left(x, Q^{2}\right)=\int_{x}^{1} \frac{d y}{y} b\left(x, y ; Q^{2}, Q_{0}^{2}\right) \bar{h}_{L}\left(y, Q_{0}^{2}\right)
$$


where,

$$
b\left(x, y ; Q^{2}, Q_{0}^{2}\right)=a(L)\left(\frac{x}{y}\right)^{p-1} \sum_{i=0}\left(\ln \frac{y}{x}\right)^{i+\rho-1} \frac{\mathcal{C}_{i}(L)}{\Gamma(i+\rho)}
$$

is the evolution kernel used in eq (31). It has been gained by using the additional relation

$$
\lim _{\substack{N, k \rightarrow \infty \\ k / N \rightarrow x}} \frac{(N+1) !}{k !} \sum_{l=0}^{N-k} \frac{(-1)^{l}}{l !(N-k-l) !} \frac{y^{k+l}}{(k+l+p)^{i+\rho}}=\frac{\theta(y-x)}{\Gamma(i+\rho) y}\left(\frac{x}{y}\right)^{p-1}\left(\ln \frac{y}{x}\right)^{i+\rho-1}
$$

For the numerical results presented in sections 4 and 5 we have verified the stability of this evolution procedure by varying the undetermined parameter $p$ in eq (71).

\section{Tensor charges in the NJL chiral soliton model}

The conventional definition of the nucleon tensor charges reads

$$
\left\langle N\left|\bar{\Psi} \sigma_{\mu \nu} \Psi\right| N\right\rangle=\Gamma_{T}^{S} \bar{u} \sigma_{\mu \nu} u \quad \text { and } \quad\left\langle N\left|\bar{\Psi} \sigma_{\mu \nu} \tau^{3} \Psi\right| N\right\rangle=\Gamma_{T}^{V} \bar{u} \sigma_{\mu \nu} \tau^{3} u
$$

Here $N$ again denotes the nucleon state. Note that both, the quark wave-function $\Psi$ as well as the nucleon spinor $u$, are vectors in flavor space. Momentum labels have been omitted as the charges are defined at zero momentum transfer. Within the NJL chiral soliton model these charges can be extracted using standard techniques [14]: first, sources conjugated to the quark bilinears $\bar{\Psi} \sigma_{\mu \nu} \Psi$ and $\bar{\Psi} \sigma_{\mu \nu} \tau^{3} \Psi$ are added to the Lagrangian (四). Subsequently the bosonized and regularized action is expanded to linear order in both the sources and the angular velocities $\Omega$ (11). The coefficients of the source terms then provide the charge operators in the space of the collective coordinates $A$, which are defined in eq (10). The corresponding matrix elements can be straightforwardly evaluated with the means provided in appendix B. Finally one obtains within the proper-time regularization

$$
\begin{aligned}
& \Gamma_{T}^{S}=\frac{N_{C}}{4 \alpha^{2}} 1 \\
&\left.+\operatorname{sgn}\left(\epsilon_{\mathrm{val}}\right)\right) \sum_{\nu \neq \text { val }} \frac{\left\langle\mathrm{val}\left|\tau^{3}\right| \nu\right\rangle\left\langle\nu\left|\beta \Sigma_{3} \tau^{3}\right| \mathrm{val}\right\rangle}{\epsilon_{\mathrm{val}}-\epsilon_{\nu}} \\
&+\frac{N_{C}}{4 \alpha^{2}} \sum_{\mu \nu} f_{\mu \nu}(\Lambda)\left\langle\mu\left|\tau^{3}\right| \nu\right\rangle\left\langle\nu \beta \Sigma_{3} \tau^{3} \mid \mu\right\rangle \\
& \Gamma_{T}^{V}=-\frac{N_{C}}{6}\left(1+\operatorname{sgn}\left(\epsilon_{\mathrm{val}}\right)\right)\left\langle\operatorname{val}\left|\beta \Sigma_{3} \tau^{3}\right| \mathrm{val}\right\rangle \\
&+\frac{N_{C}}{6} \sum_{\mu}\left\langle\mu\left|\beta \Sigma_{3} \tau^{3}\right| \mu\right\rangle \operatorname{sgn}\left(\epsilon_{\mu}\right) \operatorname{erfc}\left(\left|\frac{\epsilon_{\mu}}{\Lambda}\right|\right) .
\end{aligned}
$$


Here $|\mu\rangle$ denote the eigenstates of the static Dirac-Hamiltonian (8) and $\epsilon_{\mu}$ are the corresponding eigenvalues. Again $\mid$ val $\rangle$ refers to the distinct valence quark level. The regulator function in the isoscalar piece $(79)$ is identical to the one entering the moment of inertia, $c f$. eq (57). Those pieces containing the factor $\left(1+\operatorname{sgn}\left(\epsilon_{\mathrm{val}}\right)\right)$ are the valence contributions shown separately in table 1 .

As noted in section 5 we have omitted $1 / N_{C}$ suppressed contributions to the isovector part $\Gamma_{T}^{V}$ which in the related case of the axial current violate PCAC. 


\section{REFERENCES}

[1] J. Ralston and D. E. Soper, Nucl. Phys. B152 (1979) 109.

[2] R. L. Jaffe, X. Jin, and J. Tang, Phys. Rev. Lett. 80 (1998) 1166;

R. L. Jaffe, "Can Transversity Be Measured?", hep-ph/9710465.

[3] For a review see e.g. R. L. Jaffe, "The Spin Structure of the Nucleon", (Erice lectures) hep-ph/9602236 and references therein.

[4] R. L. Jaffe and X. Ji, Phys. Rev. Lett. 67 (1991) 552; Nucl. Phys. B375 (1992) 527.

[5] M. Stratmann, Z. Phys. C60 (1993) 763.

[6] V. Barone, T. Calarco, and A. Drago, Phys. Lett. B390 (1997) 287;

V. Barone, Phys. Lett. B409 (1997) 499.

[7] S. Scopetta and V. Vento, "A Quark Model Analysis of the Transversity Distribution", hep-ph/9706413.

[8] S. Scopetta and V. Vento, "A quark model analysis of the transversity distribution: Next to leading order evolution", hep-ph/9707250.

[9] Y. Kanazawa and Y. Koike, Phys. Lett. B403 (1997) 357.

[10] P. V. Pobylitsa and M. V. Polyakov, Phys. Lett. B389 (1996) 350.

[11] H. Weigel, Int. J. Mod. Phys. A11 (1996) 2419 and references therein.

[12] T. H. R. Skyrme, Proc. R. Soc. 127 (1961) 260;

For reviews see: G. Holzwarth and B. Schwesinger, Rep. Prog. Phys. 49 (1986) 825;

I. Zahed and G. E. Brown, Phys. Rep. 142 (1986) 481.

[13] G. S. Adkins, C. R. Nappi, and E. Witten, Nucl. Phys. B228 (1983) 552.

[14] R. Alkofer, H. Reinhardt, and H. Weigel, Phys. Rep. 265 (1996) 139.

[15] D. Ebert and H. Reinhardt, Nucl. Phys. B271 (1986) 188.

[16] Y. Nambu and G. Jona-Lasinio, Phys. Rev. 122 (1961) 345; 124 (1961) 246.

[17] H. Weigel, L. Gamberg, and H. Reinhardt, Mod. Phys. Lett. A11 (1996) 3021; Phys. Lett. B399 (1997) 287.

[18] H. Weigel, L. Gamberg, and H. Reinhardt, Phys. Rev. D55 (1997) 6910.

[19] H. Reinhardt, Nucl. Phys. A503 (1989) 825.

[20] H. Reinhardt and R. Wünsch, Phys. Lett. B215 (1988) 577; B230 (1989) 93;

T. Meißner, F. Grümmer, and K. Goeke, Phys. Lett. B227 (1989) 296;

R. Alkofer, Phys. Lett. B236 (1990) 310.

[21] A. W. Schreiber, A. I. Signal, and A. W. Thomas, Phys. Rev. D44 (1991) 2653.

[22] R. T. Cahill, C. D. Roberts, and J. Praschifka, Phys. Rev. D36 (1987) 2804.

[23] H. Reinhardt, Phys. Lett. B244 (1990) 316.

[24] J. Schwinger, Phys. Rev. 82 (1951) 664.

[25] D. R. Inglis, Phys. Rev. 96 (1954) 1059.

[26] J. L. Gervais, A. Jevicki, and B. Sakita, Phys. Rev. D12 (1975) 1038; Phys. Rep. 23 (1976) 281.

[27] R. L. Jaffe, Phys. Rev. D11 (1975) 1953.

[28] R. J. Hughes, Phys. Rev. D16 (1977) 622;

C. J. Benesh and G. A. Miller, Phys. Rev. D36 (1987) 1344.

[29] L. Gamberg, H. Reinhardt, and H. Weigel "Nucleon Structure Functions in the Infinite Momentum Frame", hep-ph/9707352. Int. J. Mod. Phys. A, to be published.

[30] R. L. Jaffe, Phys. Lett 93B (1980) 313; Ann. Phys. (NY) 132 (1981) 32. 
[31] V. N. Gribov and L. N. Lipatov, Sov. J. Nucl. Phys. 15 (1972) 438;

L. N. Lipatov, Sov. J. Nucl. Phys. 20 (1975) 94;

G. Altarelli, P. Nason, and G. Ridolfi, Phys. Lett. B320 (1994) 152, E:538.

[32] X. Artu and M. Mekhfi, Z. Phys. C45 (1990) 669.

[33] M. Traini, V. Vento, A. Mair, and A. Zambarda, Nucl. Phys. A614 (1997) 472.

[34] I. I. Balitskii, V. M. Braun, Y. Koike, and K. Tanaka, Phys. Rev. Lett. 77 (1996) 3078.

[35] A. Ali, V. M. Braun, and G. Hiller, Phys. Lett. B266 (1991) 117.

[36] S. Aoki, M. Doni, T. Hatsuda, and Y. Kuramshi, Phys. Rev. D56 (1997) 433;

M. Glöckeler et al., Nucl. Phys. Proc. Suppl. 53 (1997) 315.

[37] H. He and X. Ji, Phys. Rev. D52 (1995) 2960; Phys. Rev. D54 (1996) 6897.

[38] K. Suzuki and T. Shigetani, Nucl. Phys. A626 (1997) 886.

[39] H.-C. Kim, M. V. Polyakov, and K. Goeke, Phys. Rev. D53 (1996) 4718.

[40] R. Alkofer and H. Weigel, Phys. Lett. B319 (1993) 1.

[41] M. Wakamatsu and T. Kubota, Phys. Rev. D57 (1998) 5755.

[42] C. R. Dashen, E. Jenkins, and A. V. Manohar, Phys. Rev. D49 (1994) 4713.

[43] B. L. Ioffe and A. Khodjamirian, Phys. Rev. D51 (1995) 3373.

[44] J. Soffer, Phys. Rev. Lett. 74 (1995) 1292;

G. R. Goldstein, R. L. Jaffe, and X. Ji, Phys. Rev. D52 (1995) 5006.

[45] L. Gamberg, H. Reinhardt, and H. Weigel, in preparation.

[46] S. Kahana and G. Ripka, Nucl. Phys. A429 (1984) 445.

[47] H. Weigel, R. Alkofer, and H. Reinhardt, Nucl. Phys. B387 (1992) 638.

[48] R. Alkofer and H. Weigel, Comp. Phys. Com. 82 (1994) 30.

[49] A. W. Schreiber, A. W. Thomas, and J. J. Londergan, Phys. Rev. D42 (1990) 2226.

[50] A. Gonzáles-Arroyo, C. Lòpez, and F. J. Ynduráin, Nucl. Phys. B153 (1979) 161. 